\title{
Article \\ CSF1R Inhibition Combined with GM-CSF Reprograms Macrophages and Disrupts Protumoral Interplays with AML Cells
}

\author{
Tatiana Smirnova ${ }^{*}+{ }^{+}$, Caroline Spertini ${ }^{\dagger}$ and Olivier Spertini ${ }^{*}$ (D)
}

check for updates

Citation: Smirnova, T.; Spertini, C.; Spertini, O. CSF1R Inhibition Combined with GM-CSF Reprograms Macrophages and Disrupts Protumoral Interplays with AML Cells. Cancers 2021, 13, 5289. https:// doi.org/10.3390/cancers13215289

Academic Editor: Christian Buske

Received: 3 September 2021

Accepted: 15 October 2021

Published: 21 October 2021

Publisher's Note: MDPI stays neutral with regard to jurisdictional claims in published maps and institutional affiliations.

Copyright: (c) 2021 by the authors. Licensee MDPI, Basel, Switzerland. This article is an open access article distributed under the terms and conditions of the Creative Commons Attribution (CC BY) license (https:// creativecommons.org/licenses/by/ $4.0 /)$.
Service and Central Laboratory of Hematology, Centre Hospitalier Universitaire Vaudois, University of Lausanne, 1011 Lausanne, Switzerland; caroline.spertini@chuv.ch

* Correspondence: tatiana.smirnova@chuv.ch (T.S.); olivier.spertini@chuv.ch (O.S.)

+ These authors contributed equally to the study and are regarded as joint first authors on this manuscript.

Simple Summary: Acute myeloid leukemia is a blood disease whose long-term treatment is not satisfactory due to frequent $(>50 \%)$ relapse despite complete initial remission. By adopting protumoral properties, macrophages in the leukemia cell microenvironment may promote resistance to treatment leading to relapse. Our goal was to assess whether macrophages in contact with leukemia cells have an impact on blast survival and sensitivity to chemotherapy. We observed that leukemia cells can educate macrophages to support their survival and proliferation and reduce their sensitivity to therapy, as long as the CSF1 receptor remains active and they are in close contact. By inhibiting the activity of the CSF1 receptor, in the presence of GM-CSF, we could modify the macrophage phenotype and increase blast apoptosis and sensitivity to treatment. Our results indicate that leukemia therapies should not only target blasts but also their microenvironment and specifically macrophages and their receptor for CSF1.

Abstract: Relapse is a major issue in acute myeloid leukemia (AML) and while the contribution of gene mutations in developing drug resistance is well established, little is known on the role of macrophages (MФs) in an AML cell microenvironment. We examined whether myeloblasts could educate $M \Phi$ s to adopt a protumoral orientation supporting myeloblast survival and resistance to therapy. Flow cytometry analyses demonstrated that M2-like CD163 ${ }^{+} \mathrm{M} \Phi$ s are abundantly present, at diagnosis, in the bone marrow of AML patients. We showed that myeloblasts, or their conditioned medium, polarize monocytes to M2-like CD163 ${ }^{+} \mathrm{M} \Phi$ s, induce the secretion of many protumoral factors, and promote myeloblast survival and proliferation as long as close intercellular contacts are maintained. Importantly, pharmacologic inhibition of the CSF1 receptor (CSF1R), in the presence of GM-CSF, reprogrammed M $\Phi$ polarization to an M1-like orientation, induced the secretion of soluble factors with antitumoral activities, reduced protumoral agonists, and promoted the apoptosis of myeloblasts interacting with MФs. Furthermore, myeloblasts, which became resistant to venetoclax or midostaurin during their interplay with protumoral $\mathrm{CD} 163^{+} \mathrm{M} \Phi \mathrm{s}$, regained sensitivity to these targeted therapies following CSF1R inhibition in the presence of GM-CSF. These data reveal a crucial role of $\mathrm{CD}_{163^{+}} \mathrm{M} \Phi$ interactions with myeloblasts that promote myeloblast survival and identify CSF1R inhibition as a novel target for AML therapy.

Keywords: AML; macrophages; M-CSF; CSF1 receptor; GM-CSF; CD163; microenvironment; orientation; drug resistance; cytokines

\section{Introduction}

AML is a very aggressive disease with poor long-term survival [1]. Seventy to ninety percent of adult patients under the age of 60 achieve complete remission; however, 50-70\%, with intermediate or high genetic risk, relapse within 5 years. The European LeukemiaNet (ELN) 2017 recommendations for AML treatment are presently based on risk stratification 
by genetics and also consider the possibility to target the AML microenvironment as novel therapies [2].

Chemotherapy and hematopoietic stem cell transplantation most often allow the achievement of sustained complete remission. Moreover, targeted therapies improve the AML response to chemotherapy and favorably affect survival [3]. However, they remain restricted to a small number of AML categories. Over time and regardless of the treatment, the tumor microenvironment strongly contributes to promoting the growth and survival of drug-resistant subclones, leading to relapse [4,5]. Several clinical studies have demonstrated that heavy tumor infiltration by $M \Phi s$ is of poor prognosis: tumor-associated macrophages (TAMs) promote tumor cell growth, resistance to treatment, and escape from immune surveillance $[4,6]$.

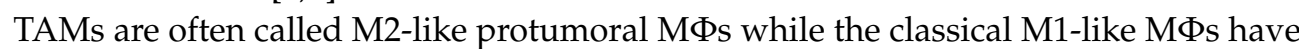
inflammatory, antitumor properties $[7,8]$. However, $M \Phi$ plasticity is complex $[9,10]$, and the M1/M2 nomenclature does not correspond to observations made in tumors, where mixed phenotype MФs, expressing both M1 and M2 markers, are observed. In the tumor microenvironment, malignant cells activate and educate $\mathrm{M} \Phi \mathrm{s}$, which secrete cytokines and promote angiogenesis, matrix remodeling, metastasis, immunosuppression, cancer cell survival, and drug resistance [9,11-13].

Tumor escape from immune surveillance is further promoted by the expression of the "do not eat me" signal, CD47 [11,14] and the increased expression of programmed cell death protein-1 (PD1) by tumor-infiltrating T-cells and of its ligand PD-L1 (programmed death-ligand 1) by TAMs [15]. While the involvement of TAMs in the solid cancer microenvironment is established [16,17], little information is available on their role in human AML. In mice, heterogeneity in M $\Phi$ polarization was observed in an MLL-AF9 AML model $[18,19]$. Myeloblasts can recruit MФs to support their proliferation in bone marrow (BM) and spleen [20].

The scavenger receptor CD163 is a marker of TAMs with protumoral characteristics $[7,12,13]$. Several studies reported that CD163 is a poor prognosis marker in solid tumors [13] and in AML [21]. CD163 expression appears to be required to allow mouse and human $M \Phi$ s to adopt a protumoral phenotype and support malignant cell proliferation. Thus, in a mouse model of sarcoma, tumor growth was abrogated in CD163 deficient mice [22]. CD163-expressing TAMs may contribute to create an immunosuppressive microenvironment. In melanoma, the depletion of $\mathrm{CD} 163^{+} \mathrm{M} \Phi$ s promotes tumor infiltration by activated T lymphocytes and tumor regression [7].

Targeting TAM polarization is a novel important therapeutic approach in aggressive cancers $[16,23]$. The colony-stimulating factor-1 (CSF-1, also called M-CSF), which controls the survival, proliferation, differentiation, and polarization of $M \Phi$ s by binding to its receptor, the CSF1R, is a promising therapeutic target in aggressive tumors and possibly in AML [24]. In multiple myeloma and mantle cell lymphoma, the inhibition of CSF1R can reprogram TAMs and reverse drug-resistance [25-28]. However, whether MФs contribute to AML cell proliferation and/or survival in creating a protumorigenic niche associated with a protumoral M $\Phi$ orientation is not established.

Using human AML cell lines and primary myeloblasts, we examined the impact of AML cells on allogeneic and autologous MФs. We showed that the co-culture of primary myeloblasts with healthy donor (HD) monocytes or with BM cells in their microenvironment induces a protumoral $M \Phi$ orientation (AML-M $\Phi$ ), which supports blast cell proliferation and survival. Furthermore, using CSF1R inhibitors and granulocyte-macrophage colony-stimulating factor (GM-CSF), we succeeded in reprogramming protumoral AMLMФs and in reversing drug-resistance induced by myeloblast cross-talk with MФs.

\section{Materials and Methods}

\subsection{Patient Samples}

Heparinized blood and BM samples were obtained from 42 newly diagnosed AML patients at CHUV (Centre Hospitalier Universitaire Vaudois, Lausanne, Switzerland). 
Informed consent was obtained from all subjects involved in the study. After red cell lysis with ammonium chloride, the samples were processed for leukocyte immunostaining and analysis by flow cytometry (FC) [29]. Leukemia diagnosis was based on WHO 2016 classification [30] and ELN 2017 risk stratification by genetics [2] and are indicated in Table S1. The study was conducted in accordance with the Declaration of Helsinki and approved by the Commission Cantonale d'Ethique sur la recherche sur l'être humain CER-VD (protocol 2017-01509, 9 November 2017).

\subsection{Immunophenotypic Analyzes by Flow Cytometry}

Cell proliferation, apoptosis, and immunophenotypic analyzes were performed using a Beckman Coulter Gallios flow cytometer. Patient samples were analyzed with a 10-color/24-antibody panel [29] according to ELN recommendations [31]. MФs were detached from culture dishes in phosphate-buffered saline (PBS) containing $10 \mathrm{mM}$ ethylenediaminetetraacetic acid (EDTA), for $10 \mathrm{~min}$ at $37^{\circ} \mathrm{C}$, washed with Roswell Park Memorial Institute (RPMI) 1640 and characterized using CD45, CD14, CD163, CD80, CD206, and CSF1R; additional blast and lymphocyte markers were used for patient $\mathrm{BM}$ and peripheral blood (PB) sample and BM co-culture analyses (Table S2).

\subsection{Healthy Donor-Derived Macrophages}

The monocytic fraction was isolated by centrifuging HD PB anticoagulated with citrate phosphate dextrose-adenine 1 and diluted $1 / 2$ with PBS for $10 \mathrm{~min}$ at $1000 \mathrm{~g}$ to prepare a buffy coat. The concentrated leukocytic fraction was then centrifuged at $400 \mathrm{~g}$ for $40 \mathrm{~min}$ on a Ficoll-Paque PLUS (GE Healthcare, Dietikon, Switzerland) gradient. Mononuclear cells were collected and plated in RPMI $+10 \%$ heat-inactivated fetal bovine serum (FBS) $=$ plain medium (PM), see Figure S1 for the schematic protocol; monocyte-derived MФs exposed to $10 \mathrm{ng} / \mathrm{mL}$ recombinant human M-CSF (ImmunoTools, Friesoythe, Germany) were called MMФs and those exposed to 1000 U/mL GM-CSF (Miltenyi Biotec, Solothurn, Switzerland): GM-MФs [25,32].

After overnight culture, the wells were rinsed to deplete non-adherent cells; $>95 \%$ of adherent cells were $\mathrm{CD} 14^{+}$monocytes. The medium was changed, with the added factors, every 2 days, for 7-9 days, when CD163 expression was checked by FC. Alternatively, to obtain $\mathrm{HD}^{\mathrm{AML}}-\mathrm{M} \Phi \mathrm{s}, \mathrm{HD}$ monocytes were stimulated for 7-9 days with (1) primary myeloblast or cell line conditioned medium (CM) diluted 1:1 with PM; with (2) AML cell lines $\left(1-5 \times 10^{4} /\right.$ well $)$ or primary blasts $\left(0.5-1 \times 10^{6} /\right.$ well $)$, co-cultured in direct contact with HD monocytes; or (3) separated from MФs by a $0.4 \mu \mathrm{m}$-pore membrane insert (Transwell (TW), Falcon, Dietikon, Switzerland).

For M $\Phi$ reprogramming experiments, HD M $\Phi$ monolayers initially activated with M-CSF (M-MФ) or blast CM (HD $\left.{ }^{A M L}-M \Phi\right)$ were washed with PBS and cultured for 7-9 more days in PM containing GM-CSF and GW2580 (1 $\mu$ M, Sigma-Aldrich, Buchs, Switzerland) [25] or PLX3397 (100 nM, MedChemExpress, Luzern, Switzerland), renewed every 2 days. The change in M $Ф$ C163 expression after reprogramming was assessed by FC, as described above.

\subsection{Cell Lines and Cell Culture}

HL-60 (AML with maturation), U937 (monoblastic leukemia), NB4 (acute promyelocytic leukemia), OCI-AML3 (NPM1 mutated) cell lines were cultured in plain medium (PM) [29] and MV-4-11 (monoblastic AML with FLT3-ITD mutation) in Iscove's Modified Dulbecco Medium $+10 \%$ FBS.

\subsection{PKH Labeling and Apoptosis Detection}

Cells were labeled with $10 \mu \mathrm{M}$ PKH26 (Sigma-Aldrich) according to the manufacturer's protocol and immediately added to rinsed wells containing adherent $M \Phi$ s for assays. A fraction of the labeled cells were fixed in 1\% paraformaldehyde and used to calculate the normalized PKH mean fluorescence intensity (MFI), as PKH MFI at day 4 
divided by PKH MFI at day 0 . Blast cell apoptosis was assessed by FC, analyzing cells stained with annexin V (AnnV, eBioscience, Zug, Switzerland).

\subsection{Cleaved Caspase-3 Detection}

Cells were cultured in 100\% PM or in 75\% PM supplemented with 25\% M $48 \mathrm{~h}$, the cells were lysed, and the cell lysates were subjected to SDS-PAGE, transferred to nitrocellulose membranes, immunoblotted with anti-cleaved caspase- 3 antibody (Cell Signaling Technology) followed by horseradish peroxidase-linked goat anti-rabbit immunoglobulin G (IgG) antibody (Cell Signaling Technology), and revealed with Luminata Forte chemiluminescence (Millipore, Schaffhausen, Switzerland) [29]. As a loading control, membranes were also immunoblotted with anti- $\alpha$-tubulin antibody (Sigma-Aldrich) followed by horseradish peroxidase-conjugated sheep anti-mouse IgG antibody (Amersham, Dietikon, Switzerland) and revealed with Luminata Crescendo chemiluminescence (Millipore). Blocking antibodies were from InvivoGen (anti-IL-6) and R\&D Systems (anti-tumor necrosis factor $\alpha$ (anti-TNF- $\alpha)$ ).

\subsection{Cytokine Arrays}

The CM of the indicated leukemia cell lines, primary myeloblasts or MФs were collected and centrifuged twice at $1500 \mathrm{rpm}$ for $10 \mathrm{~min}$, twice for $10 \mathrm{~min}$ at $2500 \mathrm{rpm}$, and filtered with $0.22 \mu \mathrm{m}$ low protein binding syringe filter. $\mathrm{CM}$ was collected at day 7 , from M- or GM-MФs cultured in medium containing M- or GM-CSF, respectively. CM from reprogrammed $M \Phi s\left(R^{\mathrm{GM} / G W}-\mathrm{M} \Phi \mathrm{s}\right)$ was obtained after one week of culture with M-CSF, followed by another week in medium supplemented with GM-CSF and GW2580. $\mathrm{CM}$ from $\mathrm{M}-, \mathrm{GM}-$, and $\mathrm{R}^{\mathrm{GM}} / \mathrm{GW}_{-} \mathrm{M} \Phi$ s were obtained from at least three different $\mathrm{HD}$ and pooled for cytokine assays. The CM from primary AML blasts (mononuclear cell suspension containing $>95 \%$ blast cells) co-cultured with $M \Phi$ were also analyzed and processed as above.

Cytokine array assays were conducted according to the manufacturer's protocol (R\&D Systems, Human XL Cytokine Array kit \#ARY022B). X-ray films were digitalized with ImageScanner III (GE Healthcare) and dot pixel density was measured with ImageQuant software (Molecular Dynamics, Glattbrugg, Switzerland). The mean of duplicates was normalized to the mean pixel density of the six reference spots.

\subsection{Myeloblast Resistance to Venetoclax and Midostaurin}

HD MФs were polarized for 7 days with M-CSF and then either reprogrammed for 7 more days in medium containing GW2580 or PLX3397 and GM-CSF or kept in M-CSFcontaining medium. M $\Phi$ orientation and reprogramming was confirmed by FC. Medium was then removed, and MФs were washed with PBS. Following this, $150 \times 10^{3}$ NB4 or MV-4-11 were added to M $\Phi$ monolayers in PM with inhibitors (venetoclax from LC Laboratories, or midostaurin from Sigma-Aldrich). After $48 \mathrm{~h}$, cells were removed from the wells, stained for AnnV and/or 7-aminoactinomycin D (7-AAD), and analyzed by FC. Experiments were repeated using MФs from at least three different HD.

\subsection{Statistical Analysis}

Statistical significance of differences between groups was examined with the MannWhitney $(\mathrm{M}-\mathrm{W})$ test between two nonparametric unpaired groups, the paired $t$-test between parametric matched samples, or the Wilcoxon matched-pairs signed rank (WMP) test between nonparametric matched samples. $p$ values $<0.05$ were considered as significant.

\section{Results}

\subsection{Presence of CD163-Expressing Macrophages in BM from AML Patients}

$\mathrm{BM}$ and/or PB were obtained from 42 adult patients with newly diagnosed AML (Table S1). MФs and myeloblasts were identified by multiparameter FC with a panel of antibodies (Table S2, see Methods for details) [29,31]. BM and PB analyses showed that 
independently of ELN risk categories [2], the majority of MФs in AML patients expressed CD163 (Figure 1A), a marker frequently associated with a protumoral phenotype [18-21]. By contrast, CD80, a marker typically associated with the inflammatory phenotype and whose expression and functions remain poorly characterized in AML, was expressed at much lower frequency $[25,27]$.
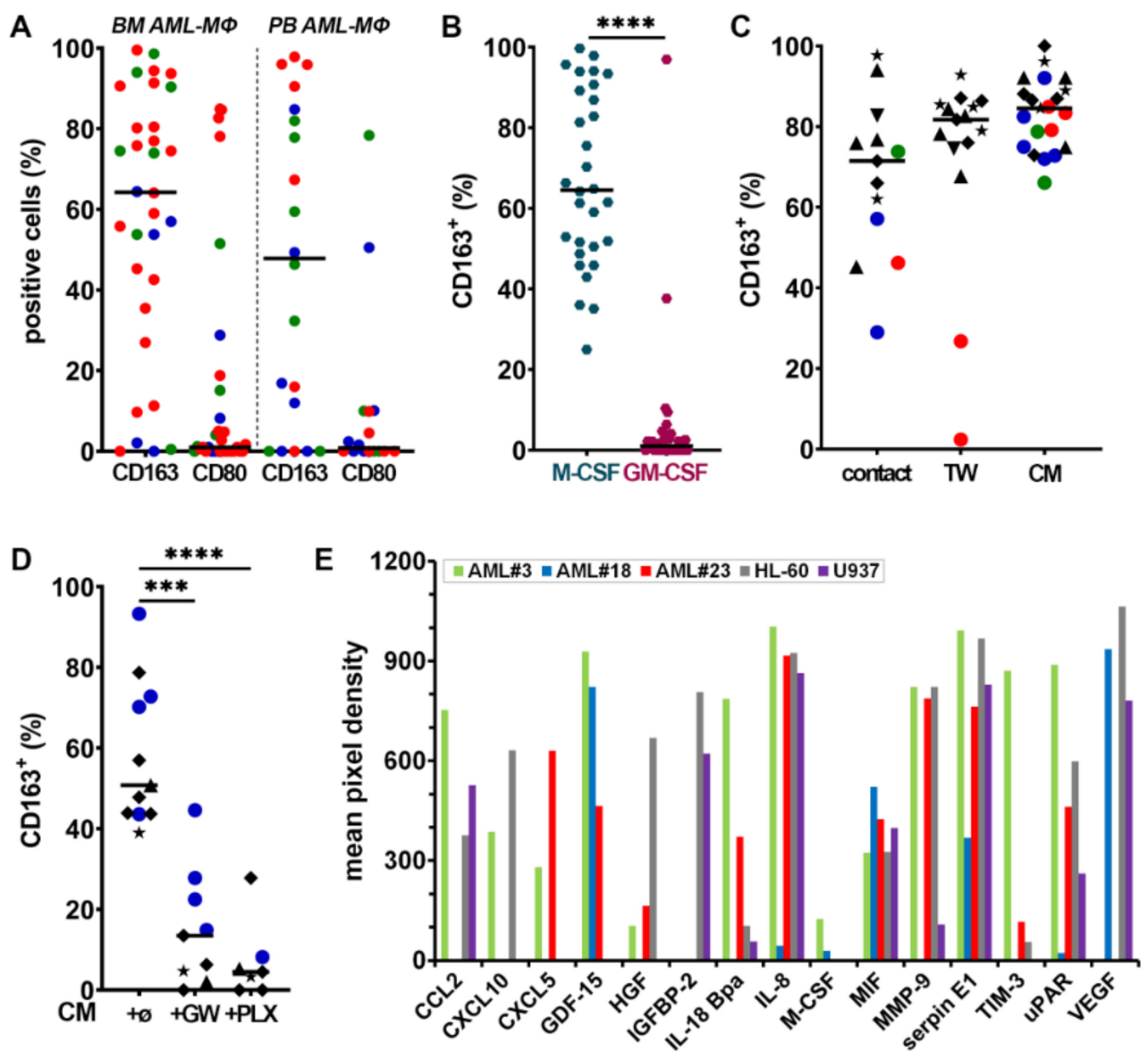

Figure 1. CD163 expression on MФs is modulated by stimuli and depends on CSF1R activity (A) Expression frequency of CD163 and CD80 in BM and/or PB of 42 AML patient MФs (within $\mathrm{CD} 14^{+} / \mathrm{CD} 45^{+}$gate) at diagnosis. Each patient is color-coded according to ELN genetic risk (green $=$ favorable, blue $=$ intermediate, and red $=$ high risk). The median is indicated by a black horizontal line. (B) Expression frequency of CD163 in HD MФs after one week of culture in PM supplemented with M- or GM-CSF, $n=30$. (C) CD163 expression allogeneic HD ${ }^{\mathrm{AML}}-\mathrm{M} \Phi$ s obtained from monocytes cultured with AML cell lines ( $\star=$ HL-60, $\boldsymbol{\Delta}=\mathrm{NB} 4, \boldsymbol{\star}=\mathrm{U} 937$, and $\boldsymbol{\nabla}=$ OCI-AML3) or primary patient blasts (dots color-coded according to genetic risk) either in direct contact or separated with a TW. AML conditioned medium (CM) was also tested. The median is indicated by a black horizontal line, $n=13-21$. (D) HD Mo were cultured for 7 days with primary patient blasts (blue) or leukemia cell lines (symbols) CM, supplemented with GW2580 (GW) or PLX3397 (PLX) before analyzing CD163 expression by FC. CM from: $\star=$ HL-60, $\boldsymbol{\Delta}=$ NB4, and $\downarrow=$ U937, blue dot = patient\#18; $n=3-6 \mathrm{HD}$. (E) Relative quantification of selected cytokines secreted by patient blasts with three different genetic risks and two leukemia cell lines. ${ }^{* * *} p<0.001$ and ${ }^{* * * *} p<0.0001$ (M-W test).

\subsection{Myeloblasts Polarize HD Monocytes to Macrophages}

We first monitored the differentiation of HD Mo into MФs by adding either M- or GM-CSF in PM for one week. Monocytes cultivated in M-CSF differentiated into M2-like CD163 ${ }^{\text {high }} \mathrm{M} \Phi$ s, whereas those cultured in GM-CSF differentiated into M1-like CD163 ${ }^{\text {low }}$ MФs (Figure 1B). To determine whether myeloblasts can polarize protumoral M $\Phi$ s and 
induce CD163 expression at their surface, we performed co-cultures of HD Mo with human AML cell lines or primary myeloblasts obtained from AML patients. After 7 days, HD Mo differentiated into MФs (HD ${ }^{\mathrm{AML}}-\mathrm{M} \Phi$ s; Figure 1C, contact), whose CD163 expression was similar to that of HD MФs cultured in medium supplemented with M-CSF.

To determine if direct contact is required for myeloblasts to polarize monocytes into MФs, they were separated with a $0.4 \mu \mathrm{m}$-pore membrane-insert (TW). CD163 expression on $\mathrm{HD}^{\mathrm{AML}}-\mathrm{M} \Phi \mathrm{s}$ was consistently observed even in the absence of direct contact (Figure 1C). Similar results were obtained by stimulating monocytes with CM from AML cell lines or primary myeloblasts. As control, the co-culture of normal hematopoietic stem progenitor cells (HSPC) with HD Mo, under the same conditions as AML cells, did not induce CD163 expression at the surface of monocytes (Figure S2A). In addition, culture in PM or with other inflammatory factors (lipopolysaccharide, TNF- $\alpha$, IL-1 $\beta$ ) did not induce CD163 expression; moreover, boiled U937 CM also failed to induce CD163 expression (Figure S3). These results suggest that soluble factors released by myeloblasts contribute to inducing $\mathrm{M} \Phi$ polarization.

Several other polarization markers were analyzed: the expression level of CD80 was expectedly higher in GM- than in M-MФs (Figure S4A) but exhibited some variability among HD ${ }^{A M L}-M \Phi s$ (Figure S4B). M- and GM-MФs exhibited variable expression of CD206 (Figure S4C), a marker typically associated with protumoral M $\Phi$ activation in different tumor types including AML [33]. CD206 was also expressed by most HD ${ }^{\mathrm{AML}}-\mathrm{M} \Phi \mathrm{s}$ (Figure S4D), both after contact with blasts or with their CM only.

As M-CSF plays a crucial role in polarizing M-MФs, we assessed whether inhibiting its receptor could prevent the up-regulation of CD163 expression induced by myeloblasts CM. Indeed, GW2580 and PLX3397, when added into CM, significantly prevented CD163 upregulation (Figure 1D), confirming a major role for CSF1R signaling in inducing CD163 expression.

To understand what factors in AML may be important in contributing to alternative MФ polarization and the induction of CD163 expression, we performed semi-quantitative cytokine array analyses using myeloblast CM from three AML patients with favorable, intermediate, or high genetic risk (patients \#3, \#18, and \#23, respectively) and from two AML cell lines (HL-60 and U937; Figure 1E). The assay can detect 105 factors, indicated in Table S3, which shows the pixel density of dots revealed on membranes illustrated in Figure S5A), some of which may contribute to promote cancer growth, cell migration, angiogenesis, and metastasis, such as CXCL5, the urokinase-type plasminogen activator receptor (uPAR), the matrix metalloproteinase 9 (MMP-9), serpin E1 [34], and the growth differentiation factor-15 (GDF-15, a novel targetable immune checkpoint [35]).

VEGF (vascular endothelial growth factor), and other angiopoietic factors, with CXCL5 [36], M-CSF [37], and CD163-expressing MФs promote angiogenesis [38]. The hepatocyte growth factor (HGF) is a key player in AML cell proliferation [24], drugresistance, and poor survival $[39,40]$. The insulin-like growth factor (IGF) and its binding protein insulin-like growth factor binding protein 2 (IGFBP-2), secreted by HL-60 and U937 cells, have important functions in promoting blast proliferation/survival and resistance to chemotherapy [41-43]. Cytokines, such as IL-18 and the IL-18-binding protein, gained increasing attention for their roles in M $\Phi$ activation syndrome [44]. Furthermore, M-CSF, macrophage migration inhibitory factor (MIF), IL-8, and CCL2 may promote a protumoral MФ polarization [25,45] contributing to forming a protective niche for leukemia stem cells [17].

CXCL10 could contribute to either a protumoral or an antitumoral activation, depending on the context [46]. The relative levels of cytokine secretion strongly differ between patients and/or leukemia cell lines, which is consistent with the heterogeneity of AML genetics, phenotypes, and biology and may contribute to the variability in the expression of markers associated to $\mathrm{HD}^{\mathrm{AML}}-\mathrm{M} \Phi$ polarization, such as CD163, CD80, and CD206. 


\subsection{HD Macrophages Promote Primary Myeloblast Survival}

Since myeloblasts can polarize monocyte-derived $М \Phi$ s (Figure 1C), we examined whether co-culturing primary human myeloblasts with HD monocytes (HD Mo) would affect blast cell survival and proliferation. Myeloblasts obtained from nine newly-diagnosed patients were cultured alone or with freshly isolated HD Mo, in direct contact or separated by a TW, for 7 days. Myeloblasts labeled with PKH26 did not reveal significant changes in proliferation when they interacted with HD Mo (T. S., personal observation, 2020). However, the co-culture of six patient cells in direct contact with HD Mo significantly improved their survival at day 7, compared to culture in plastic (Figure 2A).
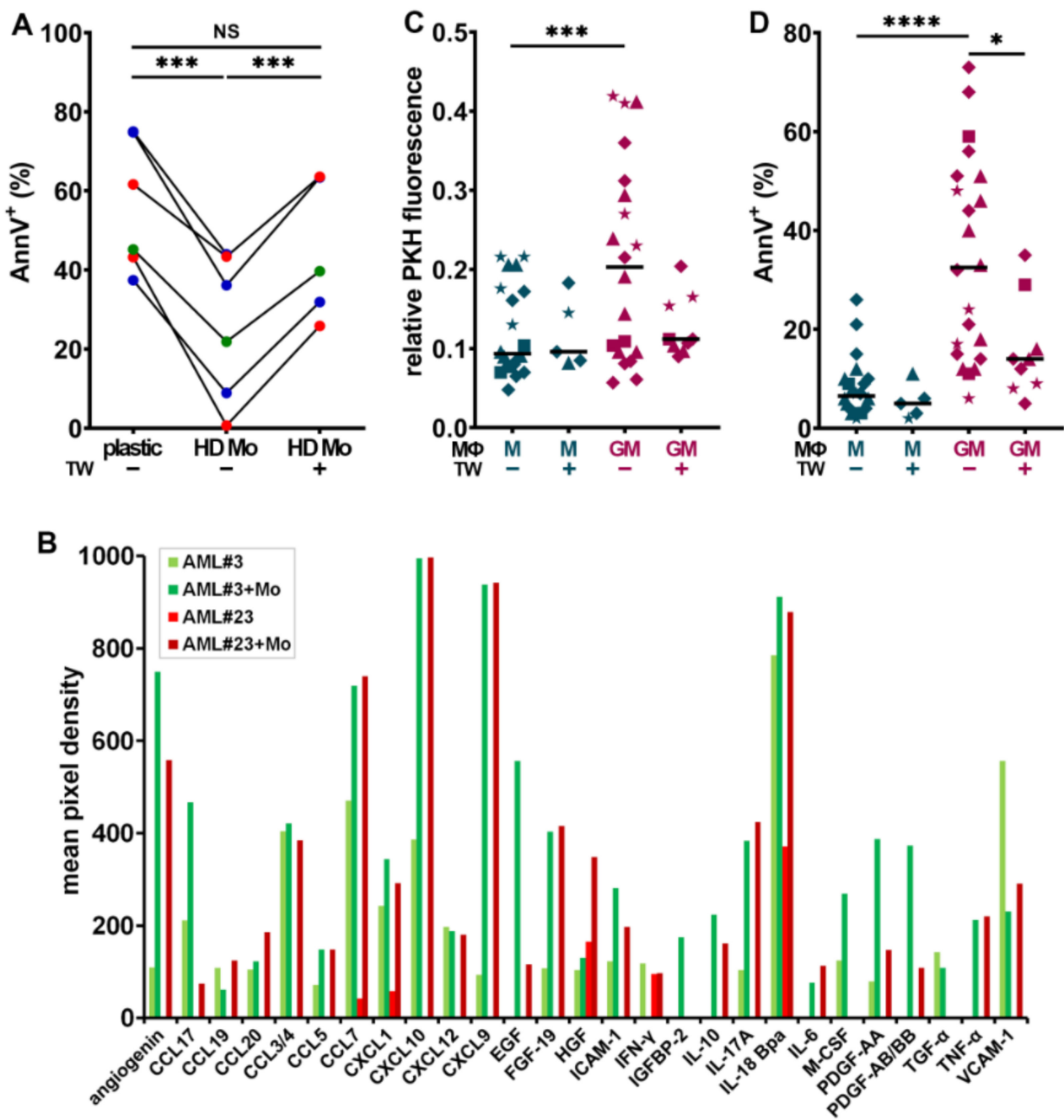

Figure 2. $\mathrm{CD}_{163^{+}} \mathrm{M} \Phi$ s support myeloblast survival and proliferation. (A) Frequency of $\mathrm{AnnV}^{+}$ AML blasts after 7 days of culture alone (plastic), or with monocytes either in direct contact (HD Mo) or separated by a TW. $n=6$ patients (\#9, 11, 17, 18, 26, and 42), color-coded according to ELN genetic risk. ${ }^{* * *} p<0.001$ (paired $t$-test). (B) Selected cytokines that were increased in CM when primary myeloblasts (favorable, green vs. high risk, red) were cultivated for 7 days with HD Mo vs. alone are illustrated. (C,D) PKH-labeled leukemia cell lines were cultured for 4 days on a monolayer of M- or GM-MФs and analyzed for proliferation (C) and survival (D). ${ }^{*} p<0.05$ (M-W test), ${ }^{* * *} p<0.001$, and ${ }^{* * *} p<0.0001$ (WMP test). $(\star=$ HL-60, $\mathbf{\square}=$ MV-4-11, $\boldsymbol{\Delta}=\mathrm{NB} 4$, and $\diamond=\mathrm{U} 937)$.

This survival advantage was dependent on direct contact with the MФs, as it was abrogated when blasts and MФs were separated by TW inserts. These observations suggest that the survival of certain sub-types of AML could be dependent on direct interactions 
with MФs. In contrast, the co-culture of normal HSPC, under the same conditions, with HD Mo did not affect their survival (Figure S2B).

The cross-talk between myeloblasts, MФs, and other components of the BM microenvironment induces the secretion of multiple proinflammatory and protumoral growth factors, which promote the remodeling of the leukemia stem cell niche and may stimulate leukemia cell proliferation and/or survival [47]. The identification of the involved soluble molecules (SM) and/or their receptors is important as they may be therapeutic targets. SM resulting from the interplay between HD Mo co-cultured with primary myeloblasts from patients \#3 and \#23 were identified after 7 days using a cytokine array (Figure S5B, Table S3).

The results were compared to those of myeloblasts cultured without monocytes (Figure S5A) and both conditions were illustrated in Figure 2B. Common and distinct characteristics are shared by AML\#3 with favorable vs. \#23 with adverse genetic risk. Several survival and proliferation factors were clearly upregulated when myeloblasts were in contact with HD Mo as opposed to cultured on plastic, such as IGFBP-2, IL-10, and epidermal growth factor (EGF) [48] (Figure 2B).

HGF was secreted at higher levels in co-culture with AML\#23. M-CSF was upregulated in co-culture with AML\#3, involved in paracrine cross-talks previously observed to drive solid tumor invasion [32,49]. Adhesion receptors VCAM-1 (vascular cell adhesion protein 1) and ICAM-1 (intercellular adhesion molecule-1) were both increased in the co-culture medium from patient $\# 23$. Several proangiogenic factors were strongly upregulated by co-culture, including angiogenin, PDGF-AA (platelet-derived growth factor), -AB/BB, and fibroblast growth factor 19 (FGF-19). Moreover, CXCL10, CXCL12, and CCL7, which may promote tumor progression, were also highly induced [32,50,51]. IL-17A, known to promote multiple myeloma cell proliferation, was highly secreted by primary myeloblasts co-cultured with MФs [52].

\section{4. $C D 163^{+}$MФs Activated with M-CSF Support Myeloblast Survival and Proliferation}

As MФs can contribute to creating a protumoral blast niche in BM microenvironment, we tested more specifically the effect of $\mathrm{M} \Phi$ orientation on blast survival and proliferation. Myeloblasts were co-cultured for 4 days with HD MФs, which had been oriented during one week in medium containing GM-CSF (GM-MФs) or M-CSF (M-M $\Phi$ s). Co-cultures were performed in PM, either in direct contact or separated with a TW.

The analysis of PKH26 dilution at day 4 showed that myeloblasts co-cultured with the $\mathrm{CD} 163^{-} \mathrm{GM}-\mathrm{M} \Phi$ s exhibited a significantly lower proliferative activity, correlated to higher PKH26 fluorescence, compared to those cultured on $\mathrm{CD}_{163^{+}} \mathrm{M}-\mathrm{M} \Phi \mathrm{s}$ (Figure 2C). Moreover, a significantly higher proportion of $\mathrm{AnnV}^{+}$myeloblasts was detected, when they were cultured in contact with GM- than with M-MФs (Figure 2D). Interestingly, in the absence of direct contact between myeloblasts and GM-MФs, blasts in TW inserts largely escaped from apoptosis. By contrast, the proportion of $\mathrm{AnnV}^{+}$blasts co-cultured with $\mathrm{M}-\mathrm{M} \Phi$ s did not significantly differ in the presence or absence of contact.

\subsection{CSF1R Inhibition and Exposure to GM-CSF Reprograms $C D 163^{+} M$ - and $H D^{A M L}-M \Phi s$}

As the upregulation of $\mathrm{CD} 163$ expression on $\mathrm{HD}^{\mathrm{AML}}-\mathrm{M} \Phi$ s can be prevented by the inhibition of CSF1R activity (Figure 1D), we assessed whether selective CSF1R tyrosine kinase inhibitors GW2580 [25] or PLX3397 [53] could modify M $\Phi$ polarization. As illustrated in Figure 3A, high CD163 expression on M-MФs was maintained after 7 additional days of culture in M-CSF (week\#2, Figure 3A). CSF1R inhibition or addition of GM-CSF during week\#2 lowered CD163 expression, with a stronger effect when GM-CSF was used in combination with either CSF1R inhibitor. In parallel, all treatments also significantly increased CD80 surface expression (Figure S6A).

The effect of $\mathrm{M}-\mathrm{M} \Phi$ reprogramming on myeloblast proliferation was assessed by comparing the proliferation of HL-60, MV-4-11, NB4, U937, and OCI-AML3 myeloblasts co-cultured for 4 days with M- vs. R-MФs. Compared to myeloblasts in direct contact with $\mathrm{CD}_{163}{ }^{+} \mathrm{M}-\mathrm{M} \Phi$ s, the co-culture with R-MФs (reprogrammed with GM-CSF combined 
with either GW2580 $=\mathrm{R}^{\mathrm{GM}} / \mathrm{GW}$, or PLX3397 $\left.=\mathrm{R}^{\mathrm{GM} / \mathrm{PLX}}\right)$, significantly slowed down their proliferation and promoted myeloblast apoptosis (Figure 3B,C). Interestingly, a significant decrease in myeloblast apoptosis was observed when they were separated from R-MФs by TW inserts. We next assessed the survival of primary myeloblasts of all three risk categories

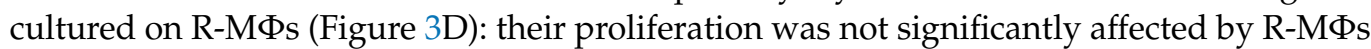
(T. S., personal observation, 2020), but their survival was strongly and significantly reduced compared to survival on M-MФs.
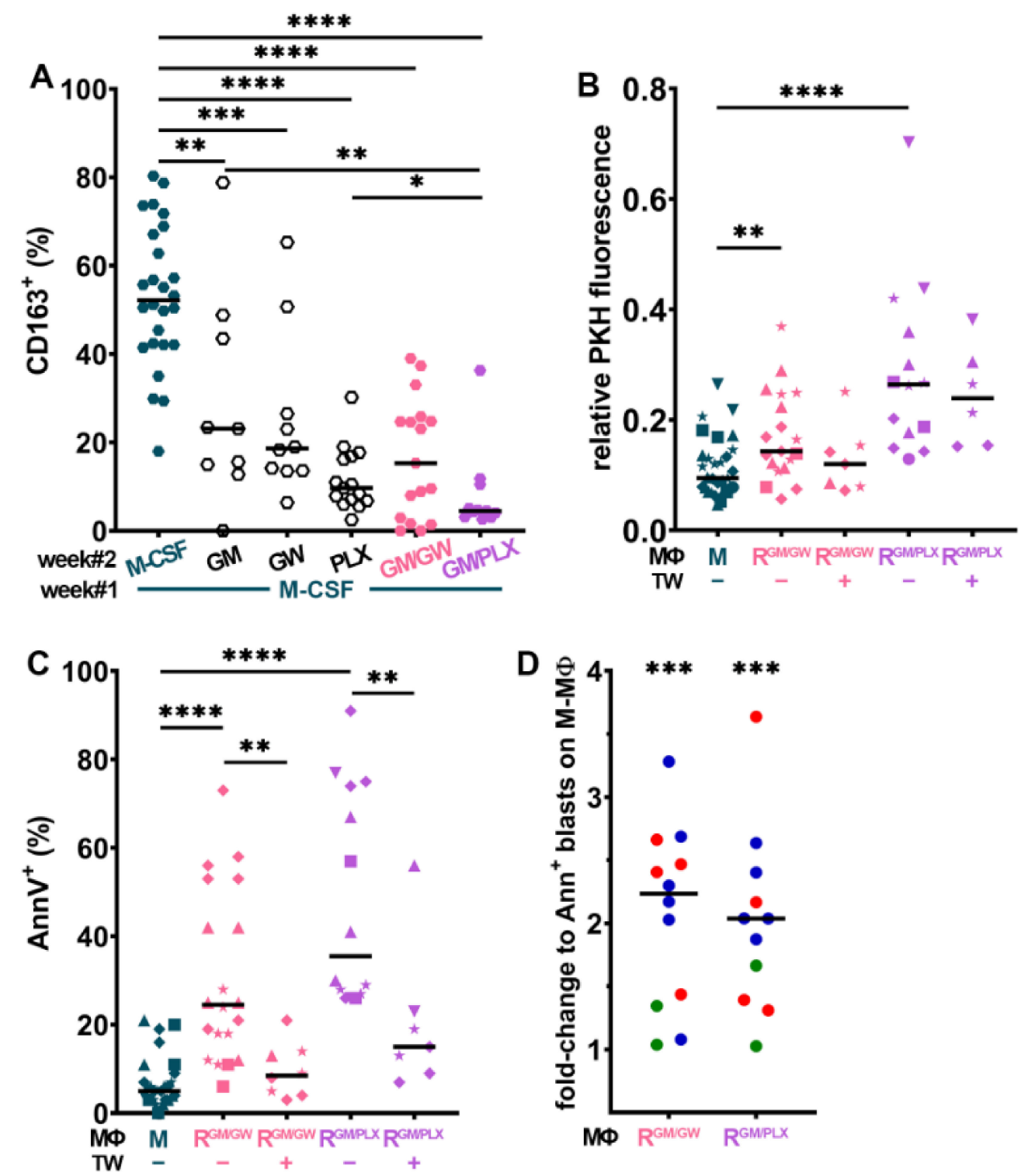

Figure 3. R-MФs induce myeloblast apoptosis. (A) Expression frequency of $\mathrm{CD}_{163^{+}} \mathrm{M} \Phi \mathrm{s}$ whose culture medium has been supplemented with M-CSF for 2 weeks or switched to reorienting medium with GM-CSF (GM) and/or GW2580 (GW) and/or PLX3397 (PLX) for one week, $n=11-26$. (B,C) PKH-labeled leukemia cell lines were cultured for 4 days on monolayers of M- or $\mathrm{R}^{\mathrm{GM} / \mathrm{GW}}$ - or R $\mathrm{R}^{\mathrm{GM} / \mathrm{PLX}}-\mathrm{M} \Phi \mathrm{s}+/$ - TW and analyzed for proliferation $(\mathbf{B})$ and survival $(\mathbf{C}), n=14-30$. $\star=$ HL-60, $\mathbf{\square}=$ MV-4-11, $\boldsymbol{\Delta}=$ NB4, $=$ U937, and $\boldsymbol{\nabla}=$ OCI-AML3. (D) Primary patient myeloblasts color-coded according to ELN genetic risk were co-cultured for 4 days on monolayers of $\mathrm{M}$ - or $\mathrm{R}^{\mathrm{GM} / \mathrm{GW}}$ - or $\mathrm{R}^{\mathrm{GM} / \mathrm{PLX}}-\mathrm{M} \Phi$ s and analyzed for survival; AnnV positivity is expressed as fold-change compared to culture on M-MФs. $n=11-12 .{ }^{*} p<0.05,{ }^{* *} p<0.01$, ${ }^{* * *} p<0.001$, and ${ }^{* * *} p<0.0001$ (using M-W test (A-C) or WMP test (D)). 
We then investigated the impact of $\mathrm{HD}^{\mathrm{AML}}-\mathrm{M} \Phi$ reprogramming: after 7 days of reorientation, single treatments significantly lowered CD163 expression, whereas the combination of GW2580 or PLX3397 with GM-CSF almost abolished it (Figure 4A); in parallel, reorientation increased CD80 expression (Figure S6B). The co-culture of HL-60, NB4, and U937 cells with $\mathrm{HD}^{\mathrm{AML}} \mathrm{R}-\mathrm{M} \Phi$ s, lowered their proliferative activity (Figure 4B) and significantly induced their apoptosis (Figure 4C). As observed with R-MФs (Figure 3C), the separation of myeloblasts from HD ${ }^{\mathrm{AML}} \mathrm{R}-\mathrm{M} \Phi$ s by a TW, protected them from apoptosis (Figure $4 \mathrm{C}$ ). These findings reveal a major role for $\mathrm{HD}^{\mathrm{AML}}-\mathrm{M} \Phi$ polarization, and most likely for adhesive interactions, in controlling myeloblast survival.
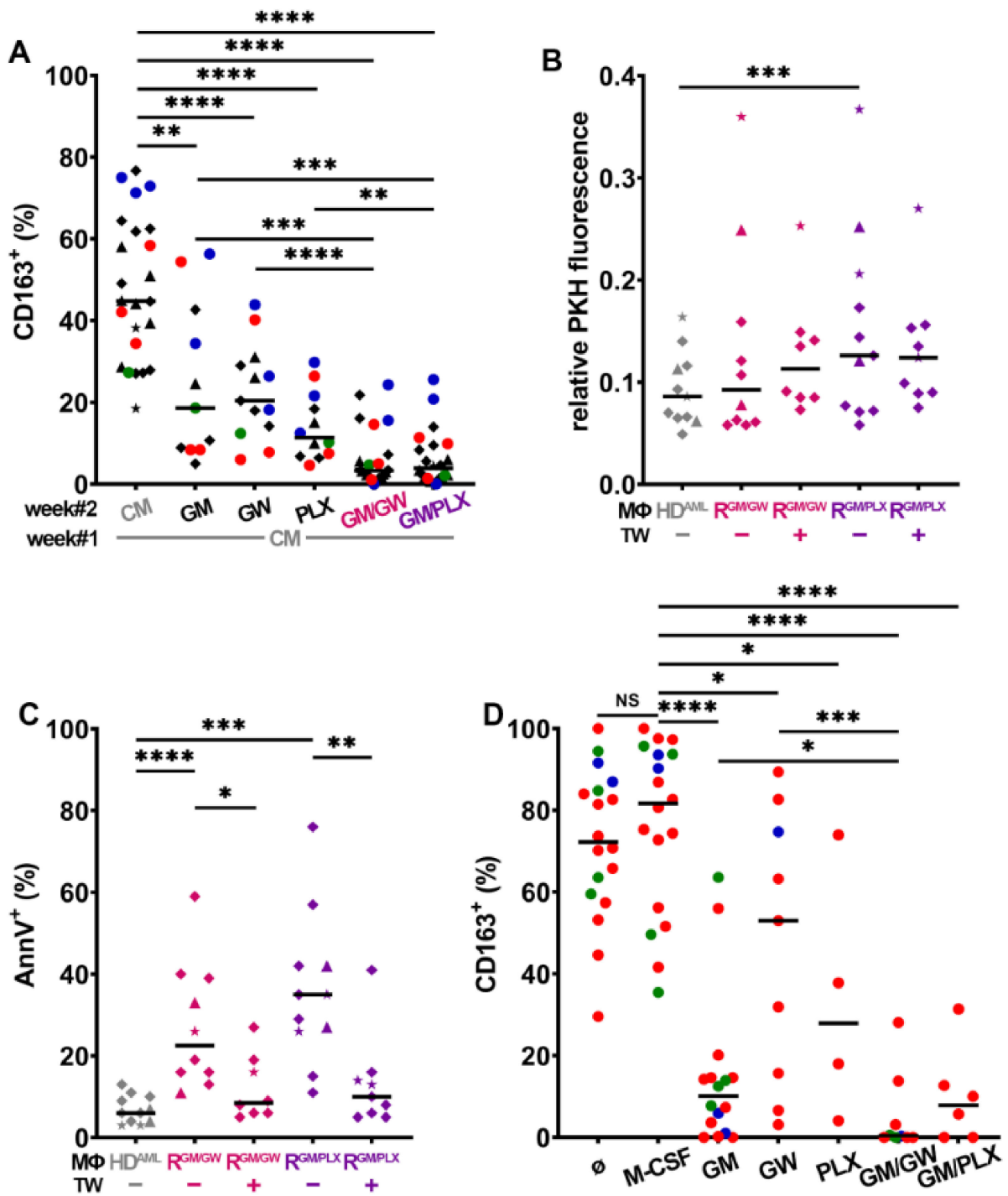

Figure 4. Reprogrammed AML macrophages induce myeloblast apoptosis. (A) After one

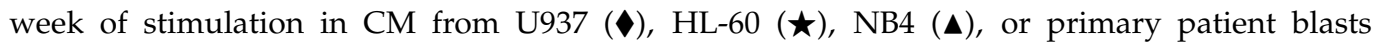
(colored dots), HD ${ }^{A M L}-M \Phi s$ were either left in the same $\mathrm{CM}$ or reoriented in $\mathrm{PM}$ with indicated supplements for one additional week and CD163 expression was analyzed by FC. $n=11-24$. (B) Myeloblast proliferation and (C) apoptosis induced after 4 days of co-culture

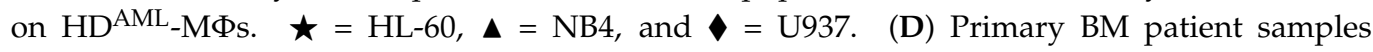
color-coded according to genetic risk were cultured for 7 days in PM + indicated supplements and analyzed by FC. (GM/GW = GM-CSF + GW2580, GM/PLX = GM-CSF + PLX3397). ${ }^{*} p<0.05,{ }^{* *} p<0.01,{ }^{* * *} p<0.001$, and ${ }^{* * * *} p<0.0001$ (using M-W test except between paired groups that were compared with WMP test). 
The impact of MФ reprogramming using CSF1R inhibitors combined to GM-CSF was then assessed on autologous primary BM MФs from AML patients. BM cells were plated at diagnosis, and MФs were co-cultured with myeloblasts, leukocytes, and stroma for 7 days under the conditions indicated in Figure 4D. Autologous BM AML-M $\Phi$ s maintained their CD163 positivity in PM, as in medium supplemented with M-CSF.

CSF1R inhibition with GW2580 was least efficient, while GM-CSF supplementation reduced CD163 expression, but not as strongly as when combined with GW2580. The combination of PLX3397 with GM-CSF also efficiently inhibited CD163 surface expression. In parallel, we monitored CD80 expression, which significantly increased with GM-CSF either alone or combined with GW2580 or PLX3397 (Figure S6C). Finally, we measured primary patient blast apoptosis in four different BM patient co-cultures in medium with M-CSF vs. reprogramming conditions; twice as many apoptotic blast cells were found in both reprogramming media compared to what was observed in M-CSF (Figure S6D).

Innate and adaptive immune checkpoint deregulation have been shown to play important roles in myeloid malignancies. We, therefore, analyzed the expression of CD47, which couples to SIRP $\alpha$ on MФs [14,54], and of PD-L1 [15]. Interestingly, PD-L1 expression was unchanged or even increased on $\mathrm{CD} 163^{\text {low }} \mathrm{HD}$ or autologous primary MФs exposed to CSF1R inhibitors, compared to CD163 ${ }^{\text {high }}$ cells cultured with M-CSF (Figure S7A,B). Further FC analyses showed that the reprogramming of autologous MФs co-cultured with primary myeloblasts did not change CD47 on myeloblasts nor SIRP $\alpha$ expression on M $\Phi$ s (Figure S7C,D).

Taken together, our results indicate that CSF1R plays a critical role in the polarization of HD M-MФs and of allogeneic $\mathrm{HD}^{\mathrm{AML}}$ - or autologous AML-MФs toward a protumoral M2-like phenotype and that inhibiting the CSF1R activity, in the presence of GM-CSF, efficiently reverses $\mathrm{M} \Phi$ orientation. This is evidenced by a down-regulation of CD163 and an increase of CD80 expression, which has a direct impact on myeloblast proliferation and survival.

\subsection{Macrophage Reprogramming Reverses Drug Resistance}

We next assessed whether M $\Phi$ orientation affects the sensitivity of myeloblasts to targeted therapy. We first examined whether $M \Phi$ polarization has an effect on myeloblast sensitivity to venetoclax, a BCL-2 inhibitor. Myeloblasts were cultured in direct contact with HD M- vs. GM-MФs (week\#1) or M- vs. R-MФs (week\#2) in presence of vehicle or escalating doses of venetoclax. After $48 \mathrm{~h}$, the percentage of live cells $\left(\mathrm{AnnV}^{-}\right.$and 7-AAD ${ }^{-}$) was measured by FC.

We observed that the co-culture of NB4 cells with M-MФs induced blast resistance to venetoclax, as compared to blasts cultured alone (T. S., personal observation, 2020). Direct contact contributed to the resistance of myeloblasts to venetoclax, as NB4 cells became significantly more sensitive to it, when they were separated from M-MФs by TW inserts (Figure 5A). By contrast, venetoclax strongly induced NB4 cell apoptosis, even at low concentrations, when blasts were cultured on GM-MФs (Figure 5A). Importantly, this was also the case when NB4 were cultured on R-MФs (reprogrammed with either GW2580 or PLX3397, and GM-CSF, $R^{G M / G W}$-, or $\mathrm{R}^{\mathrm{GM} / \mathrm{PLX}}-\mathrm{M} \Phi$ s; Figure 5B). M $\Phi$ reprogramming with GW2580 and GM-CSF also significantly sensitized OCI-AML-3 myeloblasts to venetoclax (Figure 5C).

FLT3-mutated AML have a poor prognosis [55]. To determine the impact of M $\Phi$ orientation and reprogramming on myeloblasts sensitivity to midostaurin (a FLT3 inhibitor used in AML patients [56]), we co-cultured the MLL-AF4 and FLT3-ITD mutated MV4-11 AML cells with M- vs. GM- (week\#1) or M- vs. R-MФs (week\#2). MV-4-11 cells co-cultured in direct contact with M-MФs were resistant to midostaurin (Figure 5D). In contrast, when myeloblasts were separated from M-MФs by a TW insert, midostaurin efficiently induced MV-4-11 apoptosis, suggesting an important role for cell adhesion in promoting resistance to midostaurin (Figure 5D). As observed with venetoclax, the co-culture of MV-4-11 myeloblasts on GM- or R-M $\Phi$ s strongly increased their sensitivity 
to midostaurin (Figure 5D,E). Taken together, our results suggest a critical role of $M \Phi$ orientation in promoting drug-resistance to midostaurin and venetoclax.
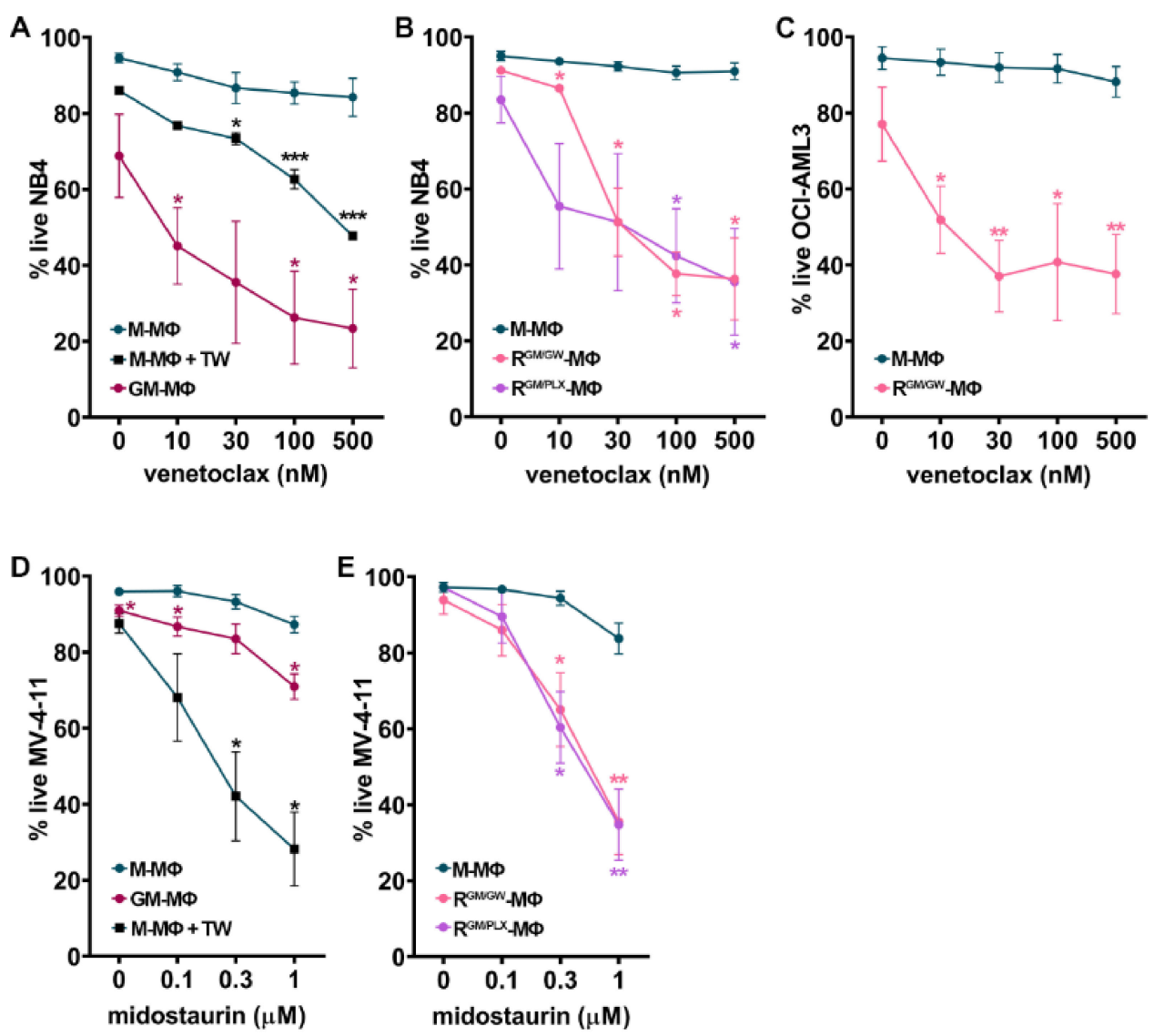

Figure 5. MФ reprogramming reverses myeloblast resistance to targeted therapy. AML cell lines were cultured with indicated $\mathrm{M} \Phi$ monolayers for $48 \mathrm{~h}$, and the frequency of live (AnnV/7-AAD-negative) cells was measured by FC in the presence of escalating doses of venetoclax (A-C), or midostaurin (D-E). (A) Frequency of live NB4 cells after culture with GM- or M-MФs either in direct contact or separated by a TW. (B) Frequency of live NB4 cells cultured with M $\Phi$ whose culture medium has been supplemented with M-CSF for 2 weeks (M-MФs) or switched to reorienting medium supplemented with GM-CSF (GM) and GW2580 (GW) or PLX3397 (PLX) (RGM/GW - or R RM/PLX $-M$ Gs) during week\#2. (C) Frequency of live OCI-AML3 as above (B) cultured with M- or RGM/GW-MФs. (D) Frequency of live (7AAD-negative) MV-4-11 cells after culture with GM- or M-MФs either in direct contact or separated by a TW. (E) The \% of live MV-4-11 cells cultured with MФs whose culture medium has been supplemented with M-CSF for 2 weeks (M-MФs) or switched to reorienting medium ( $\mathrm{R}^{\mathrm{GM} / \mathrm{GW}}$ - or $\mathrm{R}^{\mathrm{GM} / \mathrm{PLX}}$-M $\Phi \mathrm{s}$ ). Experiments were performed using monocytes isolated from 3-13 different HD. Data points represent the mean $\pm \mathrm{SEM}^{*} p<0.05,{ }^{* *} p<0.01$, and ${ }^{* *} p<0.001$ to M-MФs (M-W test).

\subsection{Factors Secreted by GM- and R-MФs Play a Role in Myeloblast Apoptosis}

In order to assess whether factors secreted by $M \Phi$ s could also have an impact on myeloblasts, HL-60, NB4, OCI-AML3, and U937 cells were cultured with MФ CM. Of the four tested cell lines, only U937 displayed sensitivity to MФ CM: after 24 h, GM-M $\Phi$ CM significantly impaired their proliferation, while $\mathrm{R}^{\mathrm{GM}} / \mathrm{GW}-\mathrm{M} \Phi \mathrm{CM}$ strongly decreased it (Figure 6A). Moreover, both $\mathrm{GM}$ - and $\mathrm{R}^{\mathrm{GM}} / \mathrm{GW}-\mathrm{M} \Phi \mathrm{CM}$ induced $>25 \%$ apoptosis in U937 cells (Figure 6B).

Effects on proliferation and apoptosis were already detectable at $6 \mathrm{~h}$ (C. S., personal observation, 2020). Moreover, after $48 \mathrm{~h}$ of incubation with $M \Phi C M$, cleaved caspase-3 was detected by western blot (Figure 6C; uncropped version Figure S8); of note, caspase-3 
cleavage was already detectable at $24 \mathrm{~h}$, but less markedly. M-MФ CM had no impact on apoptosis induction and caspase- 3 cleavage. These data indicate at least a partial contribution to apoptosis of both inflammatory/cytotoxic molecules contained in polarized GM- and $\mathrm{R}^{\mathrm{GM} / \mathrm{GW}}-\mathrm{M} \Phi \mathrm{CM}$.
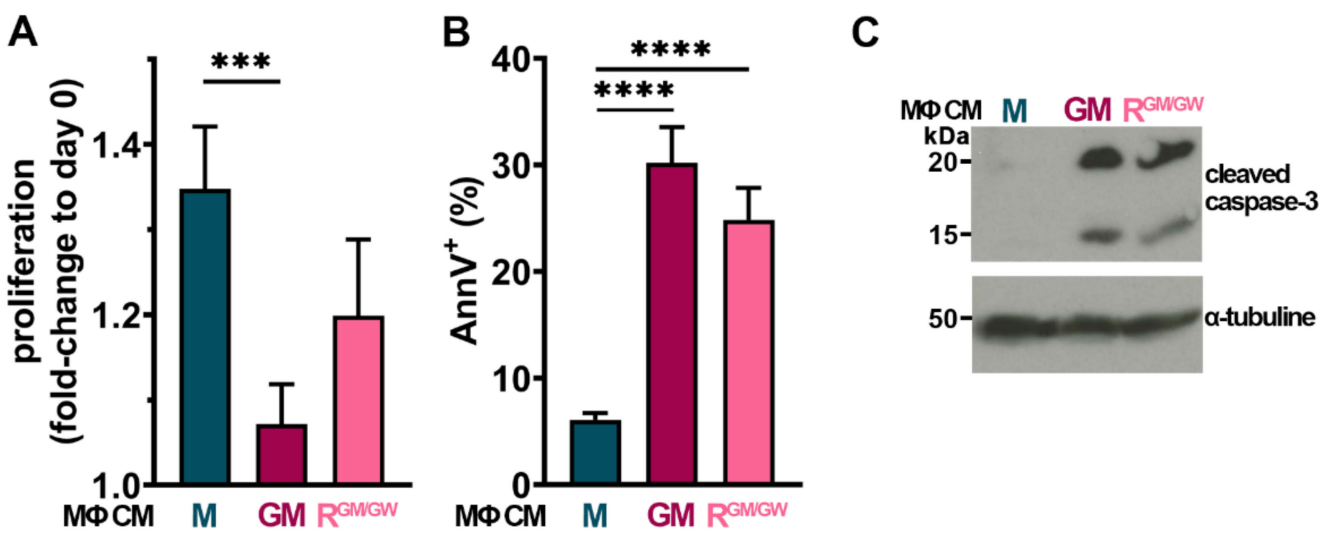

Figure 6. GM- and R- MФ CM impair U937 proliferation and induce their apoptosis. (A,B) U937 cells were cultured in PM supplemented with 25\% MФ CM and counted after $24 \mathrm{~h}, n=5-8$ (A) or analyzed by FC with AnnV staining, $n=7-13$ (B). The mean + SEM is plotted for each condition. ${ }^{* * *} p<0.001$, and ${ }^{* * * *} p<0.0001$ (M-W test). (C) Lysates from $10^{6}$ U937 cells cultivated for $48 \mathrm{~h}$ with indicated MФ CM were immunoblotted with anti-cleaved caspase- 3 antibody (upper panel) or anti- $\alpha$-tubulin (lower panel) antibody as control.

\subsection{Identification of Soluble Molecules Secreted by MФs}

To facilitate the identification of SM predominantly secreted by $\mathrm{M}_{-}, \mathrm{GM}-$, and $\mathrm{R}^{\mathrm{GM}} \mathrm{GW}$. MФs, we illustrated several relevant cytokines as their fold-decrease (Figure 7A) or -increase (Figure 7B) in GM- or R-M $\Phi$ CM relative to their quantification in M-MФ CM, while the short tables indicate the SM relative levels (data from all analyzed SM are shown in Table S3 and revealed membranes are shown in Figure S5C). M-MФs secrete prosurvival factors, such as HGF, and EGF, and high levels of VCAM-1, which has been demonstrated to play an essential role in the BM stromal compartment in mediating leukemia cell chemoresistance [57].

Other M-M $\Phi$ SM detected in the array may play a role, such as CXCL12 or IL-8 (Figure 7A and Table S3), as they contribute to support myeloblast survival and proliferation $[58,59]$ and to orient $M \Phi$ s toward immunosuppressive and proangiogenic functions [60]. Furthermore, M-M $\Phi$ reprogramming with GM-CSF and GW2580 reduced the secretion of many factors responsible for survival, angiogenesis, and cross-talk with stromal cells, for example HGF, angiogenin, IGFBP-2, IL-10, and PDGF-AB/BB. The tumor-promoting chemokine CCL7 [61] is also more abundantly secreted by M-MФs than GM- or R-MФs. CXCL9 and CXCL10, which may exhibit pro- or anti-tumoral activities, depending on the splice variant of their receptor CXCR3 [62], were reduced in CM of both GM- and R-MФs [63].

The inflammatory cytokine TNF- $\alpha$ is highly secreted in GM- and R $\mathrm{GM} / \mathrm{GW}-\mathrm{M} \Phi \mathrm{CM}$ (Figure 7B). Other factors, with context-dependent antitumoral or immune cell chemotactic activity, such as CCL5 [64], CCL17 [65], CCL3/4, CCL20 [66], CXCL1, and CCL19 [67], were also predominantly detected in CM of GM- and $\mathrm{R}^{\mathrm{GM} / \mathrm{GW}}-\mathrm{M} \Phi$ s. Compared to MФs stimulated with M-CSF, GM- and R-MФs secreted increased levels of IL-6 and IL-27, which may also contribute to inhibiting myeloblast proliferation and promoting blast apoptosis $[39,68]$.

We next determined the effect of $M \Phi$ reprogramming on soluble factor secretion resulting from the interplay between HD M $\Phi$ s co-cultured with primary myeloblasts from patient \#3 and \#23. HD Mo were first stimulated for one week with CM from patient \#3 or \#23. On day 7, after confirming the induction of CD163 expression in HD ${ }^{A M L}$. MФs, primary blasts of patients \#3 or \#23 were added to the respective $М \Phi$ monolayers 
for one more week (week\#2), in PM vs. PM supplemented with GM-CSF and GW2580 (Figure S5D). The level of each SM was illustrated as the ratio of its level in PM supplemented with GM-CSF and GW2580 divided by that secreted by co-cultures in PM (key cytokines in Figure 8).

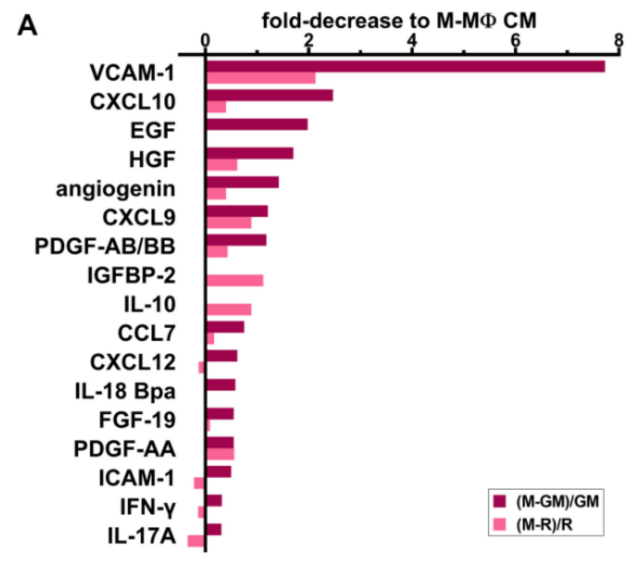

\begin{tabular}{|r|c|c|c|}
\hline$M \Phi$ CM & $M$ & GM & $R^{\text {GM/GW }}$ \\
\hline VCAM-1 & 690.3 & 79.0 & 220.3 \\
\hline CXCL10 & 920.3 & 265.4 & 659.3 \\
\hline EGF & 303.7 & 102.0 & ND \\
\hline HGF & 235.2 & 87.1 & 145.1 \\
\hline angiogenin & 714.7 & 295.7 & 511.3 \\
\hline CXCL9 & 906.2 & 409.7 & 480.3 \\
\hline PDGF-AB/BB & 455.7 & 209.1 & 317.7 \\
\hline IGFBP-2 & 448.1 & ND & 211.0 \\
\hline IL-10 & 145.8 & ND & 77.3 \\
\hline CCL7 & 520.9 & 297.3 & 444.3 \\
\hline CXCL12 & 251.7 & 155.3 & 289.4 \\
\hline IL-18 Bpa & 886.2 & 560.1 & 879.2 \\
\hline FGF-19 & 176.3 & 113.5 & 161.7 \\
\hline PDGF-AA & 154.8 & 99.8 & 99.5 \\
\hline ICAM-1 & 288.5 & 191.9 & 367.5 \\
\hline IFN-Y & 277.1 & 209.8 & 321.1 \\
\hline IL-17A & 244.5 & 186.0 & 369.3 \\
\hline & & & \\
\hline
\end{tabular}

B

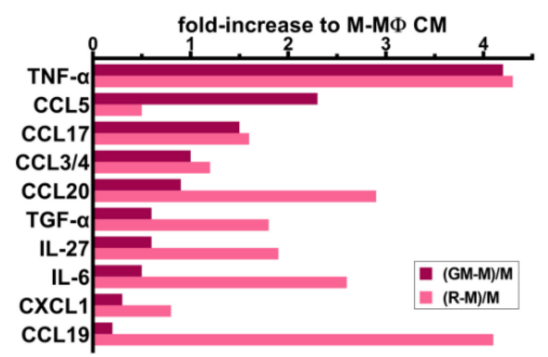

\begin{tabular}{|r|c|c|c|}
\hline$M \Phi ~ C M$ & $M$ & $G M$ & $R^{\mathrm{GM} / \mathrm{GW}}$ \\
\hline TNF- $\alpha$ & 110.7 & 574.6 & 587.4 \\
\hline CCL5 & 170.8 & 567.2 & 257.9 \\
\hline CCL17 & 380.2 & 960.2 & 995.2 \\
\hline CCL3/4 & 420.9 & 860.1 & 936.2 \\
\hline CCL20 & 200.3 & 381.3 & 790.3 \\
\hline TGF- $\alpha$ & 122.8 & 197.8 & 340.6 \\
\hline IL-27 & 104.2 & 165.9 & 297.2 \\
\hline IL-6 & 114.8 & 172.8 & 418.9 \\
\hline CXCL1 & 427.6 & 540.3 & 780.7 \\
\hline CCL19 & 141.5 & 164.2 & 727.0 \\
\hline
\end{tabular}

Figure 7. Cytokine secretion profiles differ with $M \Phi$ polarization status. HD monocytes were stimulated for 7 days with M- or GM-CSF and CM was collected and analyzed. Alternatively, CM of MФs whose culture medium has been supplemented with M-CSF for 1 week and switched to reorienting medium supplemented with GM-CSF (GM) and GW2580 (GW) for one more week was also collected. (A) A selection of cytokines is illustrated, which are decreased in GM- (dark red) and $\mathrm{R}-\mathrm{M} \Phi$ (pink) CM compared to M-M $\Phi \mathrm{CM}$, according to the formula in graph legend. (B) Selected increased cytokines in GM- (dark red) or R-M $\Phi$ (pink) CM compared to M-MФ CM are illustrated and calculated according to the formula in the graph legend. For both A and B, the relative optical density of the selected cytokines are indicated in the tables and colored from blue (low) to grey (medium) to red (high) according to their mean pixel intensity.

Consistent with analyses of GM- and R-M $\Phi$ CM shown in Figure 7, the relative levels of TNF- $\alpha$ increased in both co-cultures of AML \#3 and \#23 with HD M $\Phi$ s in the presence of GM-CSF and GW2580. The secretion of interferon $\gamma$, a key inflammatory molecule, also increased in both co-cultures under reprogramming conditions, as were CCL5, CCL17, CCL3/4, CCL19 and CCL20. IL-6 was highly secreted in CM of AML \#3 co-cultured with $\mathrm{HD}^{\mathrm{AML}}-\mathrm{M} \Phi \mathrm{s}$ in reprogramming conditions. IL-27 exhibited an opposite trend between patients. Interestingly, a number of other SM known for their protumoral activity, such as IL-10, EGF, HGF, IGFBP-2, PDGF-AB/BB, CXCL12, FGF-19, IL-18 Bpa, and VCAM1 , slightly increased or did not exhibit any significant relative change in their levels in this experiment.

\subsection{TNF- $\alpha$ Induces Apoptosis in U937 Cells}

TNF- $\alpha$ is highly increased in GM- and R-MФ CM (Figure 7B); while it could have a major function in modulating the surrounding microenvironment cross-talks, and inducing the expression and secretion of other inflammatory factors, it was shown to be cytotoxic to U937 cells [69]. We, therefore, tested the effect of escalating doses of recombinant TNF- $\alpha$ 
on U937 cells. After 24 h, we confirmed that TNF- $\alpha$ triggered apoptosis of U937 cells in a dose-dependent manner (Figure 9A). We next investigated whether adding a neutralizing antibody against TNF- $\alpha$ in MФ CM would affect the induction of apoptosis. As illustrated on Figure 9B, adding a monoclonal antibody against TNF- $\alpha$ in the CM strongly decreased apoptosis, whereas adding an antibody against IL-6, an inflammatory factor which is also increased in both $М \Phi ~ C M$, had no impact.

A

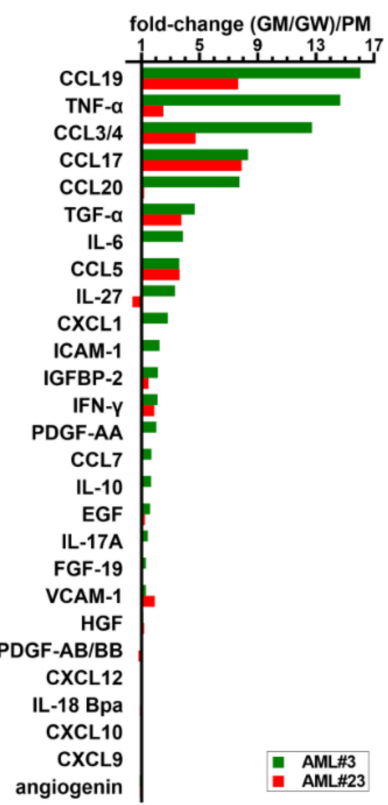

\begin{tabular}{|c|c|c|c|c|}
\hline \multirow{2}{*}{\begin{tabular}{|r|} 
week\#1 \\
week\#2 \\
\end{tabular}} & \multicolumn{2}{|c|}{ AML\#3 CM } & \multicolumn{2}{|c|}{ AML\#23 CM } \\
\hline & $\begin{array}{c}A M L \# 3 \phi \\
\text { PM }\end{array}$ & $\begin{array}{c}\text { AML\#3 } \phi \\
+\mathrm{GM} / \mathrm{GW}\end{array}$ & $\begin{array}{c}\text { AML\#23 } \varnothing \\
\text { PM }\end{array}$ & $\begin{array}{l}\text { AML\#23 } \varnothing \\
+\mathrm{GM} / \mathrm{GW}\end{array}$ \\
\hline CCL19 & 56.2 & 902.4 & 109.5 & 839.2 \\
\hline TNF- $\alpha$ & 54.8 & 804.7 & 80.4 & 201.0 \\
\hline CCL3/4 & 66.4 & 844.8 & 155.8 & 736.0 \\
\hline CCL17 & 116.0 & 966.0 & 121.1 & 956.0 \\
\hline CCL20 & 129.8 & 1006.1 & 609.6 & 714.2 \\
\hline TGF- $\alpha$ & 33.5 & 156.1 & 32.4 & 121.7 \\
\hline IL-6 & 252.3 & 974.8 & 885.5 & 912.0 \\
\hline CCL5 & 175.2 & 628.7 & 168.9 & 611.3 \\
\hline IL-27 & 60.3 & 199.1 & 184.6 & 70.4 \\
\hline CXCL1 & 260.1 & 731.1 & 659.0 & 669.8 \\
\hline ICAM-1 & 292.8 & 657.3 & 404.3 & 438.2 \\
\hline IGFBP-2 & 45.2 & 96.2 & 241.3 & 357.0 \\
\hline IFN-Y & 231.1 & 487.9 & 106.1 & 199.3 \\
\hline PDGF-AA & 148.2 & 299.2 & 367.0 & 383.0 \\
\hline CCL7 & 541.5 & 909.4 & 726.1 & 763.9 \\
\hline IL-10 & 222.6 & 369.3 & 309.8 & 335.3 \\
\hline EGF & 76.0 & 120.9 & 53.7 & 65.9 \\
\hline IL-17A & 528.0 & 754.1 & 540.4 & 507.9 \\
\hline FGF-19 & 420.8 & 546.8 & 384.5 & 422.5 \\
\hline VCAM-1 & 67.3 & 87.4 & 68.6 & 131.2 \\
\hline HGF & 168.4 & 187.9 & 241.2 & 281.8 \\
\hline PDGF-AB/BB & 372.7 & 409.1 & 493.2 & 382.9 \\
\hline CXCL12 & 567.6 & 604.4 & 616.3 & 602.3 \\
\hline IL-18 Bpa & 850.3 & 904.2 & 795.2 & 703.8 \\
\hline CXCL10 & 1092.7 & 1134.2 & 1094.1 & 1113.8 \\
\hline CXCL9 & 962.2 & 961.6 & 944.2 & 886.3 \\
\hline angiogenin & 953.3 & 829.1 & 977.0 & 861.7 \\
\hline
\end{tabular}

Figure 8. The secretion of proinflammatory cytokines is increased in co-cultures of primary AML blasts with reprogrammed $\mathrm{HD}^{\mathrm{AML}}$-M $\Phi$ s. HD Mo were stimulated for 7 days with $\mathrm{CM}$ from primary blasts from patients \#3 or \#23 and primary blasts from the same patients were then added to the respective $\mathrm{HD}^{\mathrm{AML}}-\mathrm{M} \Phi$ s with or without GM-CSF + GW2580 for 7 more days (week\#2). At the end of week\#2, CM from the four distinct co-cultures were collected and analyzed in cytokine arrays. (A) Fold change in relative secretion of selected cytokines secreted by blasts from patient \#3 (favorable risk, green bars) and \#23 (high risk, red bars) co-cultured with reprogrammed HD ${ }^{\mathrm{AML}} \mathrm{M} \Phi \mathrm{s}$ in PM containing GM-CSF and GW2580, divided by their respective relative levels in control co-cultures of AML blasts with HD ${ }^{\mathrm{AML}} \mathrm{M} \Phi$ s in PM. (B) Relative optical density of the selected cytokines illustrated in the graph colored from blue (low) to grey (medium) to red (high) according to their mean pixel density.
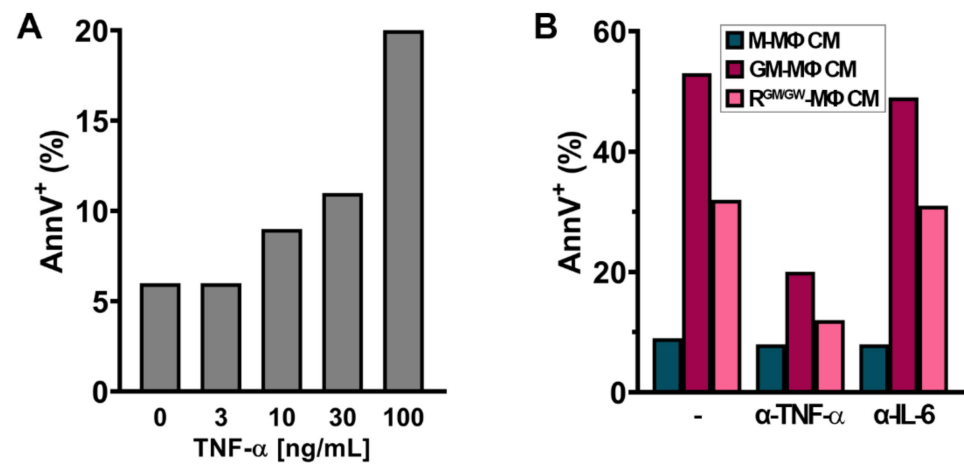

Figure 9. TNF- $\alpha$ in MФ CM can trigger apoptosis in U937 cells. (A) U937 cells were cultured for $24 \mathrm{~h}$ with increasing concentrations of TNF- $\alpha$ and apoptosis was monitored by FC. (B) U937 cells were cultured for $24 \mathrm{~h}$ with $25 \%$ indicated MФ CM and anti-TNF- $\alpha(30 \mu \mathrm{g} / \mathrm{mL})$ or anti-IL-6 $(300 \mathrm{ng} / \mathrm{mL})$ antibodies. AnnV positivity was analyzed by FC. 


\section{Discussion}

AML form a heterogeneous group of diseases, frequently of poor prognosis, with a high risk of relapse after completion of therapy. In patients with solid tumors and leukemia, the presence of TAMs has been reported as a bad prognosis factor, which may be related to the change in $M \Phi$ polarization inside tissues infiltrated by malignant cells, promoting tumor growth and survival [23]. As little information is available on AML-associated $\mathrm{M} \Phi \mathrm{s}$, we examined their impact on myeloblast survival, proliferation, and resistance to venetoclax or midostaurin.

In addition, we examined the possibility of changing the $\mathrm{M} \Phi$ orientation by inhibiting the CSF1R. Our results show that (1) BM AML M $\Phi$ s frequently express the protumoral orientation marker CD163 at diagnosis; (2) myeloblasts can educate HD MФs to express CD163 in a CSF1R-dependent manner and promote their growth and survival; (3) numerous SM are secreted that support myeloblasts proliferation, survival, migration, and angiogenesis; (4) resistance to venetoclax and midostaurin is promoted by myeloblast interactions with $\mathrm{M} \Phi \mathrm{s}$; and (5) M $\Phi$ orientation can be reprogrammed by inhibition of CSF1R combined with GM-CSF exposure, leading to the reversal of resistance to targeted therapies and myeloblast apoptosis.

$M \Phi$ s are highly plastic cells that can change their phenotype and function in response to microenvironmental stimuli, which modulate their polarization [70]. In vitro, they adopt either an antimicrobial and antitumoral activity when they are exposed to cytokines secreted by Th1 lymphocytes (such as TNF- $\alpha$ or interferon $\gamma$ ) or an anti-inflammatory phenotype promoting angiogenesis and tumor growth when they are exposed to Th2 lymphocytic cytokines (such as IL-4, -5, and -13) [71]. In tumors, depending on local cytokines secreted by malignant cells and tissue environment, TAM orientation can be shared between M1 and M2 status, with multiple phenotypes between these two extremes [15].

In the majority of AML patients, we observed that the BM and РB MФs significantly express the M2-like marker CD163 at diagnosis (Figure 1A). Interestingly, similar observations were reported in B- and T-cell acute lymphoblastic leukemia, suggesting that leukemia cells and their microenvironment may dysregulate $M \Phi$ function [72]. In a minority of AML patients, we also observed MФs expressing CD80, suggesting that a continuum of phenotypes between M1- and M2-like extremes are present at diagnosis, whose function may change in response to local signals.

The co-culture of AML cell lines or primary myeloblasts with HD Mo highly induced CD163 expression on $\mathrm{HD}^{\mathrm{AML}}$-MФs (Figure 1C), mimicking the effect of M-CSF (Figure 1B). This induction did not require direct contact between myeloblasts and $M \Phi s$ as it was also observed with exposure to AML CM, suggesting that it may depend on soluble factors, mainly cytokines [73]. However, cytokines known to induce the expression of CD163, such as IL-4, -13, and -10 [74], were not detected in AML CM (Table S3). Alternatively, we hypothesized that it may depend on CSF1/CSF1R pathway activation, as in solid cancers [15] and lymphoma [27,28], which was confirmed by the abrogation of CD163 expression induced by AML CM supplemented with GW2580 or PLX3397 (Figure 1D).

Previous observations reported a sensitivity of primary AML cells to CSF1R inhibition, in favorable-risk patients, through the secretion of cytokines, in particular HGF, by CSF1Rexpressing supportive cells [24]. Inhibiting CSF1R in order to reprogram protumoral M $\Phi$ was crucial in our experiment, but MФs were simultaneously cultured with GM-CSF to induce an M1-like polarization. GM-CSF, as a key driver of inflammatory reaction, contributes to the antitumoral effect of R-M $\Phi$ s. It may do so by enhancing the $M \Phi$ phagocytic activity, the production of reactive oxygen species, and the secretion of proinflammatory cytokines that affect T-lymphocyte response against malignant cells $[75,76]$.

M-M $\Phi$ s (Figure $3 \mathrm{~A}$ ), $\mathrm{HD}^{\mathrm{AML}}-\mathrm{M} \Phi$ s (Figure $4 \mathrm{~A}$ ), and primary autologous $\mathrm{M} \Phi$ s in their BM microenvironment (Figure 4D) were efficiently reprogrammed by CSF1R inhibition leading to a drastic decrease in CD163 expression; addition of GM-CSF potentiated this down-regulation. In co-culture assays, R-M $\Phi$ s efficiently induced primary myeloblasts and AML cell line apoptosis, but as with GM-MФs, myeloblasts were partially rescued from 
apoptosis in the absence of direct contact, suggesting a role for cell adhesion molecules. However, SM also participated in the proapoptotic effect of R-MФs. CM from GM- or R-M $\Phi$ s induced caspase-3 cleavage and the apoptosis of $>25 \%$ of U937 monoblasts.

Interestingly, mAb blocking studies indicated that TNF- $\alpha$ may contribute to inducing U937 apoptosis. High TNF- $\alpha$ levels were detected in CM of GM- and R-MФs (Figure 7B) and recombinant TNF- $\alpha$ induced U937 cell apoptosis. In myeloblasts, TNF- $\alpha$ could activate the apoptosis cascades through binding to its receptor TNFR1, FADD (Fas-associated protein with death domain) recruitment, and caspase- 8 and -3 activation or by activation of the mitochondrial pathway leading to caspase-9 and -3 activation [77].

In a mouse model of chronic lymphocytic leukemia, targeting $M \Phi$ s using an antiCSF1R mAb efficiently inhibited disease progression; interestingly, the leukemic cell death was dependent on TNF- $\alpha$ signaling and tumor microenvironment reprogramming toward an antitumoral phenotype [78]. In vivo, TNF- $\alpha$ could have not only an effect on myeloblasts but also a major impact on $\mathrm{M} \Phi$ orientation by counterbalancing the emergence of M2-like AML-M $\Phi$ s by inhibiting IL-13, and possibly IL-4, secreted by eosinophils in tumors [79].

Moreover, TNF- $\alpha$ can induce the secretion of other proinflammatory effectors, like GM-CSF, which activates interferon regulatory factor 4 (IRF4) and induces the biosynthesis of CCL17 in MФs, a cytokine loop with proinflammatory properties [80] promoting $M \Phi$ M1-like orientation and myeloblasts apoptosis. However, the CM of GM- or R-MФs had no proapoptotic effect on three other AML cell lines; this may be due to the ability of TNF- $\alpha$ to simultaneously activate pathways leading to cell apoptosis or survival and proliferation [77].

Other mechanisms may contribute in vivo to the proapoptotic effect of R-MФs. Figure 7 shows that the cytokine profile of R-M $\Phi$ s differs from that of GM-M $\Phi$ s by the secretion of higher levels of chemokines, such as CCL20, CCL19, IL-6, and IL-27. The crucial role played by CCL19 in the immune response is illustrated by its ability with IL-7 to promote tumor infiltration by $\mathrm{T}$ cells and dendritic cells and to improve the therapeutic effect of CAR-T cells against solid tumors [81] or multiple myeloma [82].

The proinflammatory molecule IL- 6 may also contribute to immune response by promoting T-cell-mediated immune defense [83] and was even reported to stimulate myeloblast differentiation by MФs [84]. Finally, blocking CSF1R in vivo inhibits monocyte recruitment into the tumor environment, leading to $M \Phi$ depletion. $M \Phi$ reprogramming induced by CSF1R inhibition may also strengthen immune defense by stimulating T-cell recruitment in a malignant cell environment [85].

Drug resistance is a major problem in relapsed/refractory AML. The combination of the BCL-2 inhibitor venetoclax with hypomethylating agents or low dose cytarabine demonstrated high initial efficiency in patients with de novo AML, who were ineligible for intensive chemotherapy [86]. However, the response to treatment is most often lost after several months, with poor survival of relapsing patients [87].

Several mechanisms of resistance to venetoclax have been reported [88] involving the up-regulation of antiapoptotic molecules Mcl-1 and Bcl-xL, mutations in genes controlling different kinase pathways, transcription factors, epigenetic modifiers, and tumor suppressors. Similarly, drug resistance affects the sensitivity of FLT3-mutated AML to midostaurin. Like for venetoclax, the mechanisms of resistance may be complex and involve the intrinsic properties of AML cells as well as of the microenvironment [89].

The ability of reprogrammed macrophages to increase the sensitivity of myeloblasts to midostaurin or venetoclax suggests a role for macrophage polarization in promoting resistance to these targeted therapies. Interestingly, close contacts between macrophages and myeloblasts were required to promote resistance to midostaurin or venetoclax, which may suggest the involvement of adhesion receptors. The presence of high soluble VCAM-1 levels in M-M $\Phi$ CM and its strong decrease in the CM of GM- and R-M $\Phi$ s may suggest the involvement of VCAM-1/CD49d, which were previously reported to trigger NF- $k B$ signaling and promote chemoresistance [57]. 


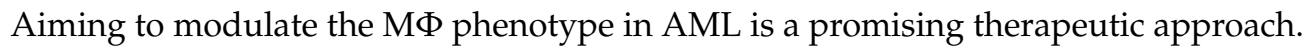
Currently, clinical development of CSF1R inhibitors, including PLX3397, for AML treatment is at the early stages [90]. The efficacy of PLX3397, a FLT3 and CSF1R inhibitor, was demonstrated in relapsed/refractory FLT3-ITD-mutated AML with a safety profile similar to that of other FLT3 inhibitors [91]. Its impact on CSF1R inhibition is unclear in relapsing FLT3-ITD AML, and its clinical activity is predominantly related to its ability to inhibit FLT3 [91]. CSF1R inhibition alone may not be sufficient to overcome resistance mechanisms in vivo [92,93], but it may represent a general approach to target the microenvironment of AML cells and may be promising when used in combination with inhibitors of the immune checkpoints, angiogenesis, or with the adoptive T-cell transfer, which are undergoing clinical investigations [94].

To reprogram human MФs in AML, combining CSF1R inhibition with GM-CSF might be more efficient for inducing an antitumoral response. It may however not be sufficient to restore $M \Phi$ phagocytic activity and antigen-presenting capacity in vivo. Indeed, PD-L1 expression is unchanged or higher on CD163 ${ }^{-} \mathrm{M} \Phi$ s exposed to CSF1R inhibitors, than on $\mathrm{CD}_{163^{+}}$cells. PD1/PD-L1 inhibition may improve the antitumoral activity of R-M $\Phi$ s and conversely, CSF1R inhibition may improve the effect of anti-PD1/PD-L1 monoclonal antibody in relapsed/refractory AML.

In addition to the T-cell immune checkpoints, CD47 is the dominant M $\Phi$ checkpoint, which is overexpressed in myeloid malignancies and inhibits phagocytosis through "do not eat me" signals upon its binding to SIRP $\alpha$ on MФs [54]. As observed for PD1/PD-L1, the reprogramming of AML-MФs co-cultured with primary myeloblasts did not change CD47 expression on myeloblasts nor SIRP $\alpha$ expression on macrophages. As the combination of the anti-CD47mAb, magrolimab, with 5-azacitidine strongly improves the antitumoral activity of MФs [54], considering a combination of CD47 blockade and CSF1R inhibitors, with 5-azacitidine, might be a way to improve the in vivo antitumoral activity of reprogrammed MФs.

\section{Conclusions}

Relapse and resistance to treatment remain major issues in the treatment of AML. We showed here that protumoral CD163 ${ }^{+} \mathrm{M} \Phi$ s predominate, at diagnosis, in the BM of AML patients and that the induction of CD163 expression on MФs by myeloblasts was dependent on CSF1R. We observed that numerous SM were secreted by MФs and myeloblasts, which promoted an M2-like M $\Phi$ orientation, leading to increased blast survival and drug resistance in the presence of close intercellular contacts. Finally, inhibiting CSF1R, in the presence of GM-CSF, reprogramed $M \Phi$ orientation, reversed the resistance to targeted therapies, and promoted myeloblast apoptosis. CSF1R inhibition may serve as a novel way to target the microenvironment of myeloblasts and improve the efficiency of AML therapy.

Supplementary Materials: The following are available online at https:/ / www.mdpi.com/article/10.3 390/cancers13215289/s1, Figure S1: Schematic protocol of MФ differentiation process, Figure S2: HSPC do not upregulate CD163 expression on MФs, Figure S3: Inflammatory or inactivated factors do not upregulate CD163 expression on HD MФs, Figure S4: CD80 and CD206 are frequently expressed by HD and $\mathrm{HD}^{\mathrm{AML}}-\mathrm{M} \Phi \mathrm{s}$, Figure S5: Autoradiographies of cytokine array membranes used for densitometry, Figure S6: CD80 expression is increased in R-MФs, Figure S7: PD-L1 is increased on R-MФs, while SIRP $\alpha$ is unchanged, Figure S8: Uncropped western blot of Figure 6C, Table S1: FAB, WHO 2016 and ELN 2017 genetic risk classifications from included patients, Table S2: Antibodies for FC, Table S3: Relative quantification of all cytokines from membranes depicted on Figure S5.

Author Contributions: Conceptualization, T.S. and O.S.; methodology, C.S., T.S. and O.S.; investigation, T.S. and C.S.; project administration, T.S.; Validation, C.S., T.S. and O.S.; resources, T.S. and O.S.; data curation, C.S. and T.S.; writing-review and editing, C.S., T.S. and O.S.; funding acquisition, T.S. All authors have read and agreed to the published version of the manuscript.

Funding: This research was supported by a grant from Fond'Action contre le cancer and the Foundation Dr. Henri Dubois-Ferrière Dinu Lipatti. 
Institutional Review Board Statement: The study was conducted according to the guidelines of the Declaration of Helsinki, and approved by the Ethics Committee of CHUV (protocol 2017-01509, 9 November 2017).

Informed Consent Statement: Informed consent was obtained from all subjects involved in the study. Data Availability Statement: Data is contained within the article or Supplementary Materials.

Acknowledgments: We thank the patients and the clinical diagnostic laboratory of hematology for providing samples after diagnostic immunophenotyping.

Conflicts of Interest: The authors declare no conflict of interest.

\section{References}

1. Burnett, A.K.; Hills, R.K.; Russell, N. Twenty five years of UK trials in acute myeloid leukaemia: What have we learned? Br. J. Haematol. 2020, 188, 86-100. [CrossRef] [PubMed]

2. Dohner, H.; Estey, E.; Grimwade, D.; Amadori, S.; Appelbaum, F.R.; Buchner, T.; Dombret, H.; Ebert, B.L.; Fenaux, P.; Larson, R.A.; et al. Diagnosis and management of AML in adults: 2017 ELN recommendations from an international expert panel. Blood 2017, 129, 424-447. [CrossRef] [PubMed]

3. Morita, K.; Wang, F.; Jahn, K.; Hu, T.; Tanaka, T.; Sasaki, Y.; Kuipers, J.; Loghavi, S.; Wang, S.A.; Yan, Y.; et al. Clonal evolution of acute myeloid leukemia revealed by high-throughput single-cell genomics. Nat. Commun. 2020, 11, 5327. [CrossRef] [PubMed]

4. Son, B.; Lee, S.; Youn, H.; Kim, E.; Kim, W.; Youn, B. The role of tumor microenvironment in therapeutic resistance. Oncotarget 2017, 8, 3933-3945. [CrossRef]

5. Gurnari, C.; Pagliuca, S.; Visconte, V. Deciphering the Therapeutic Resistance in Acute Myeloid Leukemia. Int. J. Mol. Sci. 2020, 21, 8505. [CrossRef]

6. Ruffell, B.; Coussens, L.M. Macrophages and therapeutic resistance in cancer. Cancer Cell 2015, 27, 462-472. [CrossRef]

7. Etzerodt, A.; Tsalkitzi, K.; Maniecki, M.; Damsky, W.; Delfini, M.; Baudoin, E.; Moulin, M.; Bosenberg, M.; Graversen, J.H.; Auphan-Anezin, N.; et al. Specific targeting of CD163(+) TAMs mobilizes inflammatory monocytes and promotes T cell-mediated tumor regression. J. Exp. Med. 2019, 216, 2394-2411. [CrossRef]

8. Mills, C.D. M1 and M2 Macrophages: Oracles of Health and Disease. Crit. Rev. Immunol. 2012, 32, 463-488. [CrossRef]

9. Qian, B.Z.; Pollard, J.W. Macrophage diversity enhances tumor progression and metastasis. Cell 2010, 141, 39-51. [CrossRef]

10. Murray, P.J. Macrophage Polarization. Annu. Rev. Physiol. 2017, 79, 541-566. [CrossRef]

11. Cassetta, L.; Fragkogianni, S.; Sims, A.H.; Swierczak, A.; Forrester, L.M.; Zhang, H.; Soong, D.Y.H.; Cotechini, T.; Anur, P.; Lin, E.Y.; et al. Human Tumor-Associated Macrophage and Monocyte Transcriptional Landscapes Reveal Cancer-Specific Reprogramming, Biomarkers, and Therapeutic Targets. Cancer Cell 2019, 35, 588-602.e10. [CrossRef]

12. Neubert, N.J.; Schmittnaegel, M.; Bordry, N.; Nassiri, S.; Wald, N.; Martignier, C.; Tille, L.; Homicsko, K.; Damsky, W.; Maby-El Hajjami, H.; et al. T cell-induced CSF1 promotes melanoma resistance to PD1 blockade. Sci. Transl. Med. 2018, 10 , eaan3311. [CrossRef]

13. Komohara, Y.; Jinushi, M.; Takeya, M. Clinical significance of macrophage heterogeneity in human malignant tumors. Cancer Sci. 2014, 105, 1-8. [CrossRef]

14. Takizawa, H.; Manz, M.G. Macrophage tolerance: CD47-SIRP- $\alpha$-mediated signals matter. Nat. Immunol. 2007, 8, 1287-1289. [CrossRef]

15. Noy, R.; Pollard, J.W. Tumor-associated macrophages: From mechanisms to therapy. Immunity 2014, 41, 49-61. [CrossRef]

16. Cassetta, L.; Kitamura, T. Macrophage targeting: Opening new possibilities for cancer immunotherapy. Immunology 2018, 155, 285-293. [CrossRef]

17. Mantovani, A.; Marchesi, F.; Malesci, A.; Laghi, L.; Allavena, P. Tumour-associated macrophages as treatment targets in oncology. Nat. Rev. Clin. Oncol. 2017, 14, 399-416. [CrossRef]

18. Yang, X.; Feng, W.; Wang, R.; Yang, F.; Wang, L.; Chen, S.; Ru, Y.; Cheng, T.; Zheng, G. Repolarizing heterogeneous leukemiaassociated macrophages with more M1 characteristics eliminates their pro-leukemic effects. Oncoimmunology 2018, 7, e1412910. [CrossRef]

19. Chen, C.; Wang, R.; Feng, W.; Yang, F.; Wang, L.; Yang, X.; Ren, L.; Zheng, G. Peritoneal resident macrophages in mice with MLL-AF9-induced acute myeloid leukemia show an M2-like phenotype. Ann. Transl. Med. 2021, 9, 266. [CrossRef]

20. Al-Matary, Y.S.; Botezatu, L.; Opalka, B.; Hones, J.M.; Lams, R.F.; Thivakaran, A.; Schutte, J.; Koster, R.; Lennartz, K.; Schroeder, T.; et al. Acute myeloid leukemia cells polarize macrophages towards a leukemia supporting state in a Growth factor independence 1 dependent manner. Haematologica 2016, 101, 1216-1227. [CrossRef]

21. Yan, H.; Qu, J.; Cao, W.; Liu, Y.; Zheng, G.; Zhang, E.; Cai, Z. Identification of prognostic genes in the acute myeloid leukemia immune microenvironment based on TCGA data analysis. Cancer Immunol. Immunother. 2019, 68, 1971-1978. [CrossRef]

22. Shiraishi, D.; Fujiwara, Y.; Horlad, H.; Saito, Y.; Iriki, T.; Tsuboki, J.; Cheng, P.; Nakagata, N.; Mizuta, H.; Bekki, H.; et al. CD163 Is Required for Protumoral Activation of Macrophages in Human and Murine Sarcoma. Cancer Res. 2018, 78, 3255-3266. [CrossRef]

23. Lopez-Yrigoyen, M.; Cassetta, L.; Pollard, J.W. Macrophage targeting in cancer. Ann. N. Y. Acad. Sci. 2020, 1499, 18-41. [CrossRef] 
24. Edwards, D.K.t.; Watanabe-Smith, K.; Rofelty, A.; Damnernsawad, A.; Laderas, T.; Lamble, A.; Lind, E.F.; Kaempf, A.; Mori, M.; Rosenberg, M.; et al. CSF1R inhibitors exhibit antitumor activity in acute myeloid leukemia by blocking paracrine signals from support cells. Blood 2019, 133, 588-599. [CrossRef]

25. Gutierrez-Gonzalez, A.; Martinez-Moreno, M.; Samaniego, R.; Arellano-Sanchez, N.; Salinas-Munoz, L.; Relloso, M.; Valeri, A.; Martinez-Lopez, J.; Corbi, A.L.; Hidalgo, A.; et al. Evaluation of the potential therapeutic benefits of macrophage reprogramming in multiple myeloma. Blood 2016, 128, 2241-2252. [CrossRef]

26. Wang, Q.; Liu, F.; Liu, L. Prognostic significance of PD-L1 in solid tumor: An updated meta-analysis. Medicine 2017, 96, e6369. [CrossRef]

27. Papin, A.; Tessoulin, B.; Bellanger, C.; Moreau, A.; Le Bris, Y.; Maisonneuve, H.; Moreau, P.; Touzeau, C.; Amiot, M.; PellatDeceunynck, C.; et al. CSF1R and BTK inhibitions as novel strategies to disrupt the dialog between mantle cell lymphoma and macrophages. Leukemia 2019, 33, 2442-2453. [CrossRef]

28. Valero, J.G.; Matas-Cespedes, A.; Arenas, F.; Rodriguez, V.; Carreras, J.; Serrat, N.; Guerrero-Hernandez, M.; Yahiaoui, A.; Balague, O.; Martin, S.; et al. The receptor of the colony-stimulating factor-1 (CSF-1R) is a novel prognostic factor and therapeutic target in follicular lymphoma. Leukemia 2021, 35, 2635-2649. [CrossRef]

29. Spertini, C.; Baïsse, B.; Bellone, M.; Gikic, M.; Smirnova, T.; Spertini, O. Acute Myeloid and Lymphoblastic Leukemia Cell Interactions with Endothelial Selectins: Critical Role of PSGL-1, CD44 and CD43. Cancers 2019, 11, 1253. [CrossRef]

30. Swerdlow, S.; Campo, E.; Harris, N.; Jaffe, E.; Pileri, S.; Stein, H.; Thiele, J. WHO Classification of Tumours of Haematopoietic and Lymphoid Tissues; IARC Press: Lyon, France, 2016.

31. Bene, M.C.; Nebe, T.; Bettelheim, P.; Buldini, B.; Bumbea, H.; Kern, W.; Lacombe, F.; Lemez, P.; Marinov, I.; Matutes, E.; et al. Immunophenotyping of acute leukemia and lymphoproliferative disorders: A consensus proposal of the European LeukemiaNet Work Package 10. Leukemia 2011, 25, 567-574. [CrossRef]

32. Hernandez, L.; Smirnova, T.; Kedrin, D.; Wyckoff, J.; Zhu, L.; Stanley, E.R.; Cox, D.; Muller, W.J.; Pollard, J.W.; Van Rooijen, N.; et al. The EGF/CSF-1 paracrine invasion loop can be triggered by heregulin beta1 and CXCL12. Cancer Res. 2009, 69, 3221-3227. [CrossRef] [PubMed]

33. Xu, Z.J.; Gu, Y.; Wang, C.Z.; Jin, Y.; Wen, X.M.; Ma, J.C.; Tang, L.J.; Mao, Z.W.; Qian, J.; Lin, J. The M2 macrophage marker CD206: A novel prognostic indicator for acute myeloid leukemia. Oncoimmunology 2020, 9, 1683347. [CrossRef] [PubMed]

34. Fayard, B.; Bianchi, F.; Dey, J.; Moreno, E.; Djaffer, S.; Hynes, N.E.; Monard, D. The serine protease inhibitor protease nexin-1 controls mammary cancer metastasis through LRP-1-mediated MMP-9 expression. Cancer Res. 2009, 69, 5690-5698. [CrossRef] [PubMed]

35. Wischhusen, J.; Melero, I.; Fridman, W.H. Growth/Differentiation Factor-15 (GDF-15): From Biomarker to Novel Targetable Immune Checkpoint. Front. Immunol. 2020, 11, 951. [CrossRef] [PubMed]

36. Zhang, W.; Wang, H.; Sun, M.; Deng, X.; Wu, X.; Ma, Y.; Li, M.; Shuoa, S.M.; You, Q.; Miao, L. CXCL5/CXCR2 axis in tumor microenvironment as potential diagnostic biomarker and therapeutic target. Cancer Commun. 2020, 40, 69-80. [CrossRef]

37. Kubota, Y.; Takubo, K.; Shimizu, T.; Ohno, H.; Kishi, K.; Shibuya, M.; Saya, H.; Suda, T. M-CSF inhibition selectively targets pathological angiogenesis and lymphangiogenesis. J. Exp. Med. 2009, 206, 1089-1102. [CrossRef]

38. Kobori, T.; Hamasaki, S.; Kitaura, A.; Yamazaki, Y.; Nishinaka, T.; Niwa, A.; Nakao, S.; Wake, H.; Mori, S.; Yoshino, T.; et al. Interleukin-18 Amplifies Macrophage Polarization and Morphological Alteration, Leading to Excessive Angiogenesis. Front. Immunol. 2018, 9, 334. [CrossRef]

39. Récher, C. Clinical Implications of Inflammation in Acute Myeloid Leukemia. Front. Oncol. 2021, 11, 623952. [CrossRef]

40. Sanchez-Correa, B.; Bergua, J.M.; Campos, C.; Gayoso, I.; Arcos, M.J.; Banas, H.; Morgado, S.; Casado, J.G.; Solana, R.; Tarazona, R. Cytokine profiles in acute myeloid leukemia patients at diagnosis: Survival is inversely correlated with IL-6 and directly correlated with IL-10 levels. Cytokine 2013, 61, 885-891. [CrossRef]

41. Li, T.; Forbes, M.E.; Fuller, G.N.; Li, J.; Yang, X.; Zhang, W. IGFBP2: Integrative hub of developmental and oncogenic signaling network. Oncogene 2020, 39, 2243-2257. [CrossRef]

42. Kühnl, A.; Kaiser, M.; Neumann, M.; Fransecky, L.; Heesch, S.; Radmacher, M.; Marcucci, G.; Bloomfield, C.D.; Hofmann, W.K.; Thiel, E.; et al. High expression of IGFBP2 is associated with chemoresistance in adult acute myeloid leukemia. Leuk. Res. 2011, 35, 1585-1590. [CrossRef]

43. Doepfner, K.T.; Spertini, O.; Arcaro, A. Autocrine insulin-like growth factor-I signaling promotes growth and survival of human acute myeloid leukemia cells via the phosphoinositide 3-kinase/Akt pathway. Leukemia 2007, 21, 1921-1930. [CrossRef]

44. Girard-Guyonvarc'h, C.; Palomo, J.; Martin, P.; Rodriguez, E.; Troccaz, S.; Palmer, G.; Gabay, C. Unopposed IL-18 signaling leads to severe TLR9-induced macrophage activation syndrome in mice. Blood 2018, 131, 1430-1441. [CrossRef]

45. Smirnova, T.; Bonapace, L.; MacDonald, G.; Kondo, S.; Wyckoff, J.; Ebersbach, H.; Fayard, B.; Doelemeyer, A.; Coissieux, M.M.; Heideman, M.R.; et al. Serpin E2 promotes breast cancer metastasis by remodeling the tumor matrix and polarizing tumor associated macrophages. Oncotarget 2016, 7, 82289-82304. [CrossRef]

46. Vilgelm, A.E.; Richmond, A. Chemokines Modulate Immune Surveillance in Tumorigenesis, Metastasis, and Response to Immunotherapy. Front. Immunol. 2019, 10, 333. [CrossRef]

47. Schepers, K.; Campbell, T.B.; Passegue, E. Normal and leukemic stem cell niches: Insights and therapeutic opportunities. Cell Stem Cell 2015, 16, 254-267. [CrossRef] 
48. Binder, S.; Luciano, M.; Horejs-Hoeck, J. The cytokine network in acute myeloid leukemia (AML): A focus on pro- and antiinflammatory mediators. Cytokine Growth Factor Rev. 2018, 43, 8-15. [CrossRef]

49. Wyckoff, J.; Wang, W.; Lin, E.Y.; Wang, Y.; Pixley, F.; Stanley, E.R.; Graf, T.; Pollard, J.W.; Segall, J.; Condeelis, J. A paracrine loop between tumor cells and macrophages is required for tumor cell migration in mammary tumors. Cancer Res. 2004, 64, 7022-7029. [CrossRef]

50. Boimel, P.J.; Smirnova, T.; Zhou, Z.N.; Wyckoff, J.; Park, H.; Coniglio, S.J.; Qian, B.Z.; Stanley, E.R.; Cox, D.; Pollard, J.W.; et al. Contribution of CXCL12 secretion to invasion of breast cancer cells. Breast Cancer Res. 2012, 14, R23. [CrossRef]

51. Liu, Y.; Cai, Y.; Liu, L.; Wu, Y.; Xiong, X. Crucial biological functions of CCL7 in cancer. PeerJ 2018, 6, e4928. [CrossRef]

52. Prabhala, R.H.; Pelluru, D.; Fulciniti, M.; Prabhala, H.K.; Nanjappa, P.; Song, W.; Pai, C.; Amin, S.; Tai, Y.T.; Richardson, P.G.; et al. Elevated IL-17 produced by TH17 cells promotes myeloma cell growth and inhibits immune function in multiple myeloma. Blood 2010, 115, 5385-5392. [CrossRef]

53. Quail, D.F.; Joyce, J.A. Molecular Pathways: Deciphering Mechanisms of Resistance to Macrophage-Targeted Therapies. Clin. Cancer Res. 2017, 23, 876-884. [CrossRef]

54. Chao, M.P.; Takimoto, C.H.; Feng, D.D.; McKenna, K.; Gip, P.; Liu, J.; Volkmer, J.P.; Weissman, I.L.; Majeti, R. Therapeutic Targeting of the Macrophage Immune Checkpoint CD47 in Myeloid Malignancies. Front. Oncol. 2019, 9, 1380. [CrossRef]

55. Ley, T.J.; Miller, C.; Ding, L.; Raphael, B.J.; Mungall, A.J.; Robertson, A.; Hoadley, K.; Triche, T.J., Jr.; Laird, P.W.; Baty, J.D.; et al. Genomic and epigenomic landscapes of adult de novo acute myeloid leukemia. N. Engl. J. Med. 2013, 368, 2059-2074. [CrossRef]

56. Daver, N.; Schlenk, R.F.; Russell, N.H.; Levis, M.J. Targeting FLT3 mutations in AML: Review of current knowledge and evidence. Leukemia 2019, 33, 299-312. [CrossRef]

57. Jacamo, R.; Chen, Y.; Wang, Z.; Ma, W.; Zhang, M.; Spaeth, E.L.; Wang, Y.; Battula, V.L.; Mak, P.Y.; Schallmoser, K.; et al. Reciprocal leukemia-stroma VCAM-1/VLA-4-dependent activation of NF- KB mediates chemoresistance. Blood 2014, 123, 26912702. [CrossRef]

58. Abdul-Aziz, A.M.; Shafat, M.S.; Mehta, T.K.; Di Palma, F.; Lawes, M.J.; Rushworth, S.A.; Bowles, K.M. MIF-Induced Stromal PKC $\beta$ /IL8 Is Essential in Human Acute Myeloid Leukemia. Cancer Res. 2017, 77, 303-311. [CrossRef]

59. Cho, B.S.; Zeng, Z.; Mu, H.; Wang, Z.; Konoplev, S.; McQueen, T.; Protopopova, M.; Cortes, J.; Marszalek, J.R.; Peng, S.B.; et al. Antileukemia activity of the novel peptidic CXCR4 antagonist LY2510924 as monotherapy and in combination with chemotherapy. Blood 2015, 126, 222-232. [CrossRef]

60. Sanchez-Martin, L.; Estecha, A.; Samaniego, R.; Sanchez-Ramon, S.; Vega, M.A.; Sanchez-Mateos, P. The chemokine CXCL12 regulates monocyte-macrophage differentiation and RUNX3 expression. Blood 2011, 117, 88-97. [CrossRef]

61. Lee, Y.S.; Cho, Y.B. CCL7 Signaling in the Tumor Microenvironment. Adv. Exp. Med. Biol. 2020, 1231, 33-43. [CrossRef] [PubMed]

62. Urra, S.; Fischer, M.C.; Martinez, J.R.; Veliz, L.; Orellana, P.; Solar, A.; Bohmwald, K.; Kalergis, A.; Riedel, C.; Corvalan, A.H.; et al. Differential expression profile of CXCR3 splicing variants is associated with thyroid neoplasia. Potential role in papillary thyroid carcinoma oncogenesis? Oncotarget 2018, 9, 2445-2467. [CrossRef] [PubMed]

63. Ding, Q.; Lu, P.; Xia, Y.; Ding, S.; Fan, Y.; Li, X.; Han, P.; Liu, J.; Tian, D.; Liu, M. CXCL9: Evidence and contradictions for its role in tumor progression. Cancer Med. 2016, 5, 3246-3259. [CrossRef] [PubMed]

64. Marques, R.E.; Guabiraba, R.; Russo, R.C.; Teixeira, M.M. Targeting CCL5 in inflammation. Expert Opin. Ther. Targets 2013, 17, 1439-1460. [CrossRef] [PubMed]

65. Achuthan, A.; Cook, A.D.; Lee, M.C.; Saleh, R.; Khiew, H.W.; Chang, M.W.; Louis, C.; Fleetwood, A.J.; Lacey, D.C.; Christensen, A.D.; et al. Granulocyte macrophage colony-stimulating factor induces CCL17 production via IRF4 to mediate inflammation. J. Clin. Investig. 2016, 126, 3453-3466. [CrossRef]

66. Ruytinx, P.; Proost, P.; Van Damme, J.; Struyf, S. Chemokine-Induced Macrophage Polarization in Inflammatory Conditions. Front. Immunol. 2018, 9, 1930. [CrossRef]

67. Zlotnik, A.; Yoshie, O. Chemokines: A new classification system and their role in immunity. Immunity 2000, 12, 121-127. [CrossRef]

68. Chiba, Y.; Mizoguchi, I.; Furusawa, J.; Hasegawa, H.; Ohashi, M.; Xu, M.; Owaki, T.; Yoshimoto, T. Interleukin-27 Exerts Its Antitumor Effects by Promoting Differentiation of Hematopoietic Stem Cells to M1 Macrophages. Cancer Res. 2018, 78, 182-194. [CrossRef]

69. Larrick, J.W.; Wright, S.C. Cytotoxic mechanism of tumor necrosis factor-alpha. FASEB J. 1990, 4, 3215-3223. [CrossRef]

70. Sica, A.; Mantovani, A. Macrophage plasticity and polarization: In vivo veritas. J. Clin. Investig. 2012, 122, 787-795. [CrossRef]

71. Shapouri-Moghaddam, A.; Mohammadian, S.; Vazini, H.; Taghadosi, M.; Esmaeili, S.A.; Mardani, F.; Seifi, B.; Mohammadi, A.; Afshari, J.T.; Sahebkar, A. Macrophage plasticity, polarization, and function in health and disease. J. Cell. Physiol. 2018, 233, 6425-6440. [CrossRef]

72. Dander, E.; Fallati, A.; Gulic, T.; Pagni, F.; Gaspari, S.; Silvestri, D.; Cricri, G.; Bedini, G.; Portale, F.; Buracchi, C.; et al. Monocytemacrophage polarization and recruitment pathways in the tumour microenvironment of B-cell acute lymphoblastic leukaemia. Br. J. Haematol. 2021, 193, 1157-1171. [CrossRef]

73. Skytthe, M.K.; Graversen, J.H.; Moestrup, S.K. Targeting of CD163(+) Macrophages in Inflammatory and Malignant Diseases. Int. J. Mol. Sci. 2020, 21, 5497. [CrossRef]

74. Etzerodt, A.; Moestrup, S.K. CD163 and inflammation: Biological, diagnostic, and therapeutic aspects. Antioxid. Redox Signal. 2013, 18, 2352-2363. [CrossRef] 
75. Lee, K.M.C.; Achuthan, A.A.; Hamilton, J.A. GM-CSF: A Promising Target in Inflammation and Autoimmunity. Immunotargets Ther. 2020, 9, 225-240. [CrossRef]

76. Kim, I.K.; Koh, C.H.; Jeon, I.; Shin, K.S.; Kang, T.S.; Bae, E.A.; Seo, H.; Ko, H.J.; Kim, B.S.; Chung, Y.; et al. GM-CSF Promotes Antitumor Immunity by Inducing Th9 Cell Responses. Cancer Immunol. Res. 2019, 7, 498-509. [CrossRef]

77. Aggarwal, B.B.; Gupta, S.C.; Kim, J.H. Historical perspectives on tumor necrosis factor and its superfamily: 25 years later, a golden journey. Blood 2012, 119, 651-665. [CrossRef]

78. Galletti, G.; Scielzo, C.; Barbaglio, F.; Rodriguez, T.V.; Riba, M.; Lazarevic, D.; Cittaro, D.; Simonetti, G.; Ranghetti, P.; Scarfo, L.; et al. Targeting Macrophages Sensitizes Chronic Lymphocytic Leukemia to Apoptosis and Inhibits Disease Progression. Cell Rep. 2016, 14, 1748-1760. [CrossRef]

79. Kratochvill, F.; Neale, G.; Haverkamp, J.M.; Van de Velde, L.A.; Smith, A.M.; Kawauchi, D.; McEvoy, J.; Roussel, M.F.; Dyer, M.A.; Qualls, J.E.; et al. TNF Counterbalances the Emergence of M2 Tumor Macrophages. Cell Rep. 2015, 12, 1902-1914. [CrossRef]

80. Cook, A.D.; Lee, M.C.; Saleh, R.; Khiew, H.W.; Christensen, A.D.; Achuthan, A.; Fleetwood, A.J.; Lacey, D.C.; Smith, J.E.; Forster, I.; et al. TNF and granulocyte macrophage-colony stimulating factor interdependence mediates inflammation via CCL17. JCI Insight 2018, 3, e99249. [CrossRef]

81. Adachi, K.; Kano, Y.; Nagai, T.; Okuyama, N.; Sakoda, Y.; Tamada, K. IL-7 and CCL19 expression in CAR-T cells improves immune cell infiltration and CAR-T cell survival in the tumor. Nat. Biotechnol. 2018, 36, 346-351. [CrossRef]

82. Duan, D.; Wang, K.; Wei, C.; Feng, D.; Liu, Y.; He, Q.; Xu, X.; Wang, C.; Zhao, S.; Lv, L.; et al. The BCMA-Targeted FourthGeneration CAR-T Cells Secreting IL-7 and CCL19 for Therapy of Refractory/Recurrent Multiple Myeloma. Front. Immunol. 2021, 12, 609421. [CrossRef] [PubMed]

83. Li, B.; Jones, L.L.; Geiger, T.L. IL-6 Promotes T Cell Proliferation and Expansion under Inflammatory Conditions in Association with Low-Level ROR $\gamma$ t Expression. J. Immunol. 2018, 201, 2934-2946. [CrossRef] [PubMed]

84. Kawashima, T.; Murata, K.; Akira, S.; Tonozuka, Y.; Minoshima, Y.; Feng, S.; Kumagai, H.; Tsuruga, H.; Ikeda, Y.; Asano, S.; et al. STAT5 induces macrophage differentiation of M1 leukemia cells through activation of IL-6 production mediated by NF-kappaB p65. J. Immunol. 2001, 167, 3652-3660. [CrossRef] [PubMed]

85. Fujiwara, T.; Yakoub, M.A.; Chandler, A.; Christ, A.B.; Yang, G.; Ouerfelli, O.; Rajasekhar, V.K.; Yoshida, A.; Kondo, H.; Hata, T.; et al. CSF1/CSF1R Signaling Inhibitor Pexidartinib (PLX3397) Reprograms Tumor-Associated Macrophages and Stimulates T-cell Infiltration in the Sarcoma Microenvironment. Mol. Cancer Ther. 2021, 20, 1388-1399. [CrossRef] [PubMed]

86. DiNardo, C.D.; Pratz, K.; Pullarkat, V.; Jonas, B.A.; Arellano, M.; Becker, P.S.; Frankfurt, O.; Konopleva, M.; Wei, A.H.; Kantarjian, H.M.; et al. Venetoclax combined with decitabine or azacitidine in treatment-naive, elderly patients with acute myeloid leukemia. Blood 2019, 133, 7-17. [CrossRef]

87. Maiti, A.; Rausch, C.R.; Cortes, J.E.; Pemmaraju, N.; Daver, N.G.; Ravandi, F.; Garcia-Manero, G.; Borthakur, G.; Naqvi, K.; Ohanian, M.; et al. Outcomes of relapsed or refractory acute myeloid leukemia after frontline hypomethylating agent and venetoclax regimens. Haematologica 2021, 106, 894-898. [CrossRef]

88. Saliba, A.N.; John, A.J.; Kaufmann, S.H. Resistance to venetoclax and hypomethylating agents in acute myeloid leukemia. Cancer Drug Resist. 2021, 4, 125-142. [CrossRef]

89. Yang, X.; Sexauer, A.; Levis, M. Bone marrow stroma-mediated resistance to FLT3 inhibitors in FLT3-ITD AML is mediated by persistent activation of extracellular regulated kinase. Br. J. Haematol. 2014, 164, 61-72. [CrossRef]

90. Sletta, K.Y.; Castells, O.; Gjertsen, B.T. Colony Stimulating Factor 1 Receptor in Acute Myeloid Leukemia. Front. Oncol. 2021, 11, 654817. [CrossRef]

91. Smith, C.C.; Levis, M.J.; Frankfurt, O.; Pagel, J.M.; Roboz, G.J.; Stone, R.M.; Wang, E.S.; Severson, P.L.; West, B.L.; Le, M.H.; et al. A phase 1/2 study of the oral FLT3 inhibitor pexidartinib in relapsed/refractory FLT3-ITD-mutant acute myeloid leukemia. Blood Adv. 2020, 4, 1711-1721. [CrossRef]

92. Quail, D.F.; Bowman, R.L.; Akkari, L.; Quick, M.L.; Schuhmacher, A.J.; Huse, J.T.; Holland, E.C.; Sutton, J.C.; Joyce, J.A. The tumor microenvironment underlies acquired resistance to CSF-1R inhibition in gliomas. Science 2016, 352, aad3018. [CrossRef]

93. Cai, H.; Zhang, Y.; Wang, J.; Gu, J. Defects in Macrophage Reprogramming in Cancer Therapy: The Negative Impact of PD-L1/PD-1. Front. Immunol. 2021, 12, 690869. [CrossRef]

94. Ries, C.H.; Hoves, S.; Cannarile, M.A.; Ruttinger, D. CSF-1/CSF-1R targeting agents in clinical development for cancer therapy. Curr. Opin. Pharmacol. 2015, 23, 45-51. [CrossRef] 